J. DIFFERENTIAL GEOMETRY

89 (2011) 1-47

\title{
EINSTEIN SPACES AS ATTRACTORS FOR THE EINSTEIN FLOW
}

\author{
Lars Andersson \& Vincent MoncRief
}

\begin{abstract}
In this paper we prove a global existence theorem, in the direction of cosmological expansion, for sufficiently small perturbations of a family of $n+1$-dimensional, spatially compact spacetimes, which generalizes the $k=-1$ Friedmann-Lemaître-RobertsonWalker vacuum spacetime. This work extends the result from [3]. The background spacetimes we consider are Lorentz cones over negative Einstein spaces of dimension $n \geq 3$.

We use a variant of the constant mean curvature, spatially harmonic (CMCSH) gauge introduced in [2]. An important difference from the $3+1$ dimensional case is that one may have a nontrivial moduli space of negative Einstein geometries. This makes it necessary to introduce a time-dependent background metric, which is used to define the spatially harmonic coordinate system that goes into the gauge.

Instead of the Bel-Robinson energy used in [3], we here use an expression analogous to the wave equation type of energy introduced in [2] for the Einstein equations in CMCSH gauge. In order to prove energy estimates, it turns out to be necessary to assume stability of the Einstein geometry. Further, for our analysis it is necessary to have a smooth moduli space. Fortunately, all known examples of negative Einstein geometries satisfy these conditions.

We give examples of families of Einstein geometries which have nontrivial moduli spaces. A product construction allows one to generate new families of examples.

Our results demonstrate causal geodesic completeness of the perturbed spacetimes, in the expanding direction, and show that the scale-free geometry converges toward an element in the moduli space of Einstein geometries, with a rate of decay depending on the stability properties of the Einstein geometry.
\end{abstract}

\section{Introduction}

Let $M$ be a compact, connected, orientable manifold of dimension $n \geq 2$, and assume that $M$ admits a smooth Riemannian Einstein metric

Received August 3, 2009. 
$\gamma$ with negative Einstein constant. After a trivial rescaling, we can suppose that

$$
\operatorname{Ric}[\gamma]=-\frac{n-1}{n^{2}} \gamma,
$$

where $\operatorname{Ric}[\gamma]$ is the Ricci tensor of $\gamma$. With this normalization, the Lorentz cone spacetime $\bar{M}=(0, \infty) \times M$ with metric

$$
\bar{g}=-d t \otimes d t+\frac{t^{2}}{n^{2}} \gamma
$$

is globally hyperbolic and Ricci flat, i.e. a solution of the vacuum Einstein equations in dimension $n+1$, and admits a homothetic Killing field $Z=t \partial_{t}$, such that $\mathcal{L}_{Z} \bar{g}=-2 \bar{g}$. Such spacetimes are said, by virtue of this global homothety, to be continuously self-similar.

We showed in an earlier paper $[\mathbf{3}]$ that in the case $n=3$, Lorentz cone spacetimes are stable to the future. The main result of $[\mathbf{3}]$ is that for constant mean curvature (CMC) Cauchy data for the vacuum Einstein equations close to the standard data for a Lorentz cone, the maximal future Cauchy development is globally foliated by CMC Cauchy surfaces. Further, the Cauchy development is causally geodesically complete to the future, and the induced spatial metric on the CMC Cauchy surfaces converges, after a suitable rescaling, to the background metric $\gamma$.

The result in $[\mathbf{3}]$ required that the background metric $\gamma$ satisfy a nontrivial rigidity condition, namely that it allows no nontrivial, traceless Codazzi tensors. Kapovich [11, theorem 2] has proved the existence of compact hyperbolic spaces with this property; see also the discussion in $[3, \S 2.4]$. The rigidity condition corresponds to the assumption that the moduli space of flat spacetimes at the Lorentz cone $(\bar{M}, \bar{g})$ is trivial. The rigidity condition was later removed by Reiris [15].

The argument in $[\mathbf{3}]$ and also in $[\mathbf{1 5}]$ relied on Bel-Robinson type energies to control the (fully nonlinear) perturbations, and is therefore essentially restricted to the $3+1$ dimensional case; see [16], however. In this paper, our aim is to extend the analysis to general dimension. In order to do this, we introduce a new family of energies for the Einstein equations, which are not curvature-based.

1.1. Lorentz cone spacetimes. In the case $n=2$, the Einstein condition implies that $(M, \gamma)$ is a hyperbolic surface. Since other, more farreaching techniques are available in two spatial dimensions $[\mathbf{1}, \mathbf{4}, \mathbf{1 3}, \mathbf{1 4}]$, we shall here concentrate on dimensions $n \geq 3$.

When $n=3, \gamma$ is necessarily hyperbolic, i.e. has constant negative sectional curvature, and indeed hyperbolic metrics provide the most familiar special cases of negative Einstein metrics in all higher dimensions as well, but when $n \geq 4$ many examples of non-hyperbolic, negative Einstein metrics are known to exist [5].

By Mostow rigidity, hyperbolic metrics are unique up to isometry, and trivial homothetic rescalings, for all dimensions $n \geq 3$, but when 
$n=2$ there exists, for each higher genus surface, a finite dimensional Teichmüller space of non-isometric hyperbolic metrics. Nontrivial connected finite dimensional manifolds of negative Einstein metrics can also occur when $n \geq 4$, but, as we shall see below, these cannot contain a hyperbolic member, since higher dimensional hyperbolic metrics are always isolated as Einstein metrics.

When $\gamma$ is hyperbolic, as is necessarily true for $n=2$ and $n=3$, $\bar{g}$ is actually flat and indeed the spacetime $(\bar{M}, \bar{g})$ can be regarded as the quotient of the interior of the future light cone of a point in $n+1$ dimensional Minkowski space by a subgroup of the proper orthochronous Lorentz group which fixes that point. When $\gamma$ is only Einstein, however, and does not have constant curvature, the metric $\bar{g}$ is not in general flat.

If we let $\tilde{g}$ and $\tilde{K}$ denote respectively the first and second fundamental forms induced on a $t=$ constant hypersurface of $(\bar{M}, \bar{g})$, then

$$
\tilde{g}=\frac{t^{2}}{n^{2}} \gamma, \quad \tilde{K}=-t^{-1} \tilde{g} .
$$

Furthermore, the mean curvature $\tau=\operatorname{tr}_{g} K=g^{i j} K_{i j}$ is given by $\tau=$ $-n t^{-1}$ so that the hypersurfaces of constant $t$ are in fact constant mean curvature (CMC) slices labelled by the value of their mean curvature, which could be used as a time function. As $\tau$ ranges over $(-\infty, 0)$, the spaces evolve from a zero volume "big bang" to an infinite volume limit of cosmological expansion.

It is possible to prove directly, cf. [10], that any vacuum spacetime (or, by a straightforward generalization, non-vacuum spacetime obeying a suitable energy condition), which admits a compact, orientable CMC Cauchy hypersurface, and a nontrivial, proper homothetic Killing field, must in fact be a Lorentz cone spacetime of the type described above (and so, in particular, devoid of matter). In a suitable time gauge, the reduced Hamiltonian takes the value

$$
H_{\text {reduced }}=|\tau|^{n} \operatorname{Vol}(M, \tilde{g}),
$$

cf. $[\mathbf{1 0}, \S 4.1]$. The Lorentz cone spacetimes can also be characterized uniquely as critical points for the reduced Hamiltonian [10, theorem 3], when the latter is re-expressed in terms of its natural canonical variables.

1.2. Rescaled Einstein equations. A closely related characterization of these spacetimes is that they are the unique fixed points of what we call the rescaled Einstein equations. Noting that the mean curvature has dimensions (length) $)^{-1}$, adopting CMC slicing with mean curvature $\tau=\operatorname{tr}_{g} K$ as "time" and taking the spatial coordinates $\left(x^{i}\right)$ to be dimensionless, we find that the dimensions of the embedding variables of a Cauchy surface in spacetime are given by

$$
\begin{aligned}
& {\left[\tilde{g}_{i j}\right]=(\text { length })^{2}, \quad\left[\tilde{K}_{i j}\right]=\text { (length) }} \\
& {[\tilde{N}]=(\text { length })^{2}, \quad\left[\tilde{X}^{i}\right]=(\text { length }) .}
\end{aligned}
$$


We define rescaled, dimensionless variables $(g, \Sigma, N, X)$ by setting

$$
\begin{aligned}
g_{i j} & =\tau^{2} \tilde{g}_{i j}, \quad \Sigma_{i j}=\tau\left(\tilde{K}_{i j}-\frac{\tau}{n} \tilde{g}_{i j}\right), \\
N & =\tau^{2} \tilde{N}, \quad X^{i}=\tau \tilde{X}^{i},
\end{aligned}
$$

and rewrite the field equations in terms of these quantities. For a treatment using the canonical ADM variables, see [10].

When $\tau$ is taken to serve as time, all of the conventional ADM equations - constraints, evolution equations and gauge fixing equations needed to enforce the CMC slicing-become non-autonomous since the mean curvature $\tau$ appears in all of them. When these are expressed in terms of the rescaled variables, however, all of this explicit $\tau$-dependence is scaled away except for derivatives with respect to $\tau$, which take the dimensionless form $\tau \partial_{\tau}$. One can remove this final explicit $\tau$-dependence by simply defining a new, dimensionless time coordinate $T$ by

$$
T=-\ln \left(\tau / \tau_{0}\right)
$$

and reexpressing $\tau \partial_{\tau}$ as $-\frac{\partial}{\partial T}$. Note that the natural range of $T$ is $\mathbb{R}$, whereas $\tau$ only ranged over $(-\infty, 0)$. The transformed field equations are given explicitly in equations $(4.9,4.10)$, while the elliptic equations determining the rescaled lapse function and shift vector field are given in (4.11). The harmonic spatial coordinate condition that we shall impose later will not disturb the autonomous character of the field equations, but one should note that the inclusions of a cosmological constant or non-scale-invariant matter sources would disturb this character.

While autonomous field equations are not strictly essential for what we wish to do, it is convenient to begin with the simplest cases and to deal with generalizations later.

The rescaled variables for the Lorentz cone spacetimes defined above are given by

$$
\begin{aligned}
g=\gamma, \quad \Sigma=0, \\
N=n, \quad X=0 .
\end{aligned}
$$

The time independence of these quantities shows directly that they are indeed fixed points of the rescaled equations. By [10, theorem 2], they are the only fixed points of this system and, as we have mentioned, are the only solutions admitting a nontrivial proper and globally defined homothetic Killing field (namely $Z=\partial_{T}$ ).

1.3. Linearized analysis. In $[\mathbf{1 0}, \S 3]$, the linearized equations for perturbations about an arbitrary fixed point were studied. The transversetraceless (TT) perturbations can be naturally decomposed in terms of the TT eigentensors of the operator $\mathcal{L}$ defined by

$$
\mathcal{L} h_{a b}=-\Delta h_{a b}-2 R_{a c b d} h^{c d}
$$


where $\Delta=\gamma^{c d} \nabla_{c} \nabla_{d}$, with $\nabla$ the covariant derivative defined with respect to $\gamma$, and $R_{a b c d}$ the Riemann tensor of $\gamma$. The above operator is closely related to the one defined in [10, equation (3.12)], and also to the Lichnerowicz Laplacian $\Delta_{L}$, cf. equation (2.3). Note that the compatibility of this operator with the TT character of the eigentensor depends upon the fact that $\gamma$ is Einstein; see section 2 for details.

The eigenvalues $\lambda$ of $\mathcal{L}$ are all real, since $\mathcal{L}$ is self-adjoint with respect to the natural $L^{2}$ inner product. A separation of variables argument may be used to analyze the linearized, rescaled, Einstein equations (cf. section 6.3 below; see also [10]). The character of the corresponding

solution depends upon the value of $\lambda$ as follows. If $\lambda>\frac{(n-1)^{2}}{4 n^{2}}$, then the characteristic equation has a complex pair of roots with real part $-(n-$ $1) / 2$, and hence there is a universal exponential rate of decay $-(n-1) / 2$, in the time $T$. If $0<\lambda<\frac{(n-1)^{2}}{4 n^{2}}$, the characteristic equation has a pair of negative real roots. In this case we have an "anomalous" rate of decay depending on $\lambda$. In the marginal case $\lambda=\frac{(n-1)^{2}}{4 n^{2}}$, the system has a resonance. We avoid dealing directly with the marginal case by considering a slightly decreased $\lambda$. If $\lambda=0$, we would have a "neutral" mode, which does not decay. Typically, this situation corresponds to the existence of a nontrivial moduli space of Einstein metrics containing $\gamma$.

If $\lambda<0$ were to occur, the characteristic equation would have a root with positive real part and the corresponding solution would grow exponentially rather than decay. It is an open question whether any such "unstable" Einstein spaces exist. The above discussion motivates calling $\gamma$ stable if $\mathcal{L}$ has nonnegative spectrum. We shall review what is known below; cf. section 2 .

1.4. Stable Einstein spaces and moduli spaces. When $n=3$, an Einstein metric is necessarily hyperbolic (in the negative case of interest here) and Mostow rigidity excludes the possibility of deforming the hyperbolic (hence Einstein) structure. In this sense, $n=3$ is the most "rigid" dimension - a compact manifold either admits no negative Einstein structure or precisely one.

For $n>3$, Mostow rigidity still applies, but now the new possibility arises of having negative Einstein spaces that are not hyperbolic. Many families of such (negative) Einstein spaces are known to exist and whenever a chosen background metric $\gamma$ belongs to a smooth (necessarily finite dimensional modulo gauge degrees of freedom) such family, the linearized equations will always admit a corresponding (finite dimensional) space of neutral modes with $\lambda=0$. These represent the tangent space, at the given background, to the space of self-similar spacetimes. Such smooth families of self-similar spacetimes, determined by the corresponding families of negative Einstein metrics, are expected to form "center manifolds" for the dynamical system defined by the rescaled Einstein equations, and we shall see below that this is in fact the case. 
If there are no obstructions to integrating an infinitesimal Einstein deformation to a curve of Einstein structures, then the moduli space is a manifold (cf. $[\mathbf{5}, \S 12 . \mathrm{F}]$ ). We refer to such moduli spaces as integrable; cf. Definition 2.3 below. In particular, there are examples of negative Einstein spaces contained in an integrable moduli space, such that $\mathcal{L}$ has nonnegative spectrum.

One such family is given by negative Kähler-Einstein metrics; cf. section 2.5. Hyperbolic metrics in dimension $n \geq 3$ are rigid, in the sense that the moduli space of Einstein metrics is trivial, and further are strictly stable, in the sense that the spectrum of $\mathcal{L}$ is positive (see section 2.4 ). If $n=2$, however, one can show that zero is always in the spectrum of $\mathcal{L}$, since every $\mathrm{TT}$ tensor on a higher genus surface is a traceless Codazzi tensor [4]. This corresponds precisely to the presence of a full Teichmüller space of self-similar solutions to the Einstein equations to which the tangent space at any one such solution corresponds to the space of "neutral modes" defined by TT tensors. This possibility arises precisely for $n=2$ by virtue of the failure of Mostow rigidity for hyperbolic structures to hold for surfaces.

1.5. Stability of Lorentz cone spacetimes. The main result of this paper gives a nonlinear stability result for Lorentz cone metrics, which generalizes the results of $[\mathbf{3}, \mathbf{1 5}]$ from the $3+1$ dimensional to the $n+1$ dimensional case. The result requires that $\gamma$ be stable in the sense discussed in section 1.4 above, and that $\gamma$ be either rigid, or contained in an integrable moduli space of Einstein structures. For data sufficiently close to the standard data for the Lorentz cone over $\gamma$, the rescaled geometry tends in the expanding direction to a limit in the moduli space of $\gamma$. It is this fact which motivates the title of the paper. For the case of Ricci flow, stable Einstein spaces also play the role of attractors, in a sense which is closely related to the one discussed above; see [8].

The idea that (stable) Einstein spaces are attractors for the Einstein flow is motivated by the linearized analysis in [10] (see section 6.3 below), as well as by the fact that the reduced Hamiltonian $H_{\text {reduced }}$ has positive semi-definite Hessian at the Lorentz cone data exactly when $\gamma$ is stable, together with the observation that $H_{\text {reduced }}$ is monotone decreasing to the future; cf. [10].

The main result in this paper is analogous to the results of $[4,14]$, where it is shown that the rescaled geometry of the level sets of the mean curvature time function converges to a point in Teichmüller space, with the Einstein moduli space playing the same role as Teichmüller space.

The work in [3] relied on the analysis of the Einstein equations in CM$\mathrm{CSH}$ gauge, i.e. CMC time gauge with spatial harmonic coordinates. In the present work, due to the presence of nontrivial moduli spaces of Einstein background metrics, it is necessary to use a generalization of the CMCSH gauge, which allows for a time-dependent background 
metric for the spatial harmonic coordinates. The time-dependent background metric is determined by a so-called shadow metric condition, which requires that the difference between the rescaled metric $g$ and the background metric used to define the spatial harmonic gauge be $L^{2}$-orthogonal to the deformation space (or premoduli space, in the terminology of [5]). The presence of a nontrivial moduli space makes necessary some rather delicate considerations in order to prove the required energy estimates.

1.6. Overview of this paper. Section 2 gives the necessary background material on negative Einstein spaces, and introduces the notion of stability. In section 2.5 we collect some known examples of stable negative Einstein spaces with integrable moduli space. In section 3 we show that Cartesian products of stable spaces are stable, and that taking products of stable spaces with integrable moduli space yields new spaces with the same property. This allows us to construct large families of examples where our results apply.

Next, in section 4, we introduce the rescaled system of Einstein equations which will be studied, together with the shadow metric condition, that is, the gauge condition generalizing the CMCSH condition of [2] which we use in the case when the moduli space is nontrivial. Section 5 discusses the proof of local wellposedness for the rescaled Einstein equations with shadow metric gauge, based on the work in [2].

The linearized, rescaled Einstein equations are introduced and analyzed in section 6 . This section also contains an analysis of the damped oscillator equation which arises from a separation of variables of these same linearized equations. The behavior of the solutions of this equation was studied in [10]. However, for the present purposes, we need an energy argument which yields the correct decay estimates. The energy for the damped oscillator equation is analyzed in section 6.4. In section 7 , this analysis is used as the basis for a definition of energies for the full rescaled Einstein equations. The energies we use have a lot in common with the energies used for the local existence proof in CMCSH gauge; cf. [2]. Finally, section 8 gives the statement and proof of the main results of the paper.

The appendix A provides the proof of Lemma 4.3.

1.7. Preliminaries and notation. Let $M$ be a compact manifold of dimension $n \geq 2$ and let $\mathcal{M}$ denote the space of Riemannian metrics on $M$. For $g \in \mathcal{M}$, we denote by Riem, Ric, Scal, the Riemann and Ricci tensor, and the scalar curvature, respectively. We shall often use index notation, with lowercase Latin indices running over $1, \ldots, n$ and Greek indices running over $0, \ldots, n$. The index versions of Riem and Ric are $R_{a b c d}$ and $R_{a b}=R_{a c b}^{c}$, respectively. The Christoffel symbols of $g$ are denoted by $\Gamma_{m n}^{i}$. We shall sometimes indicate that a curvature tensor 
or Christoffel symbol is defined with respect to a special metric, say $\gamma$, by writing, e.g., $R[\gamma]_{a b c d}, \Gamma[\gamma]_{m n}^{i}$.

We shall often work in the context of Sobolev regular metrics. For an integer $s$, let $H^{s}$ denote the $L^{2}$ Sobolev spaces on $M$, defined with respect to some once-and-for-all given background metric. We use the notation $\|\cdot\|_{H^{s}}$ for the $H^{s}$ norm. For $s>n / 2+1$, denote by $\mathcal{M}^{s}$, $\mathcal{E}_{\alpha}^{s}$ the spaces of metrics, and Einstein metrics with Einstein constant $\alpha$, of Sobolev class $s$. For an Einstein metric, we may without loss of generality, by working in harmonic coordinates, assume that it is $C^{\infty}$ or in fact real analytic; see [9]. For most standard situations, we leave it to the reader to fill in the analytical details and drop the Sobolev index from our notation.

Acknowledgments. Both authors are grateful to the Erwin Schrödinger Institute in Vienna and the Mittag-Leffler Institute, Djursholm, Sweden for hospitality and support during the work on this paper. Moncrief also thanks l'Institute des Hautes Études Scientifiques in Bures-sur-Yvette and the Albert Einstein Institute in Golm for hospitality and support during the work on this paper. The authors thank Michael Anderson for helpful remarks.

Andersson was supported in part by the NSF, with grants DMS0407732 and DMS-0707306 to the University of Miami. Moncrief was supported in part by the NSF, with grants PHY-0354391 and PHY0647331 to Yale University.

\section{Negative Einstein Spaces}

In this section we shall review some material on negative Einstein spaces which is needed in the rest of the paper. We emphasize the notion of stability for Einstein spaces; cf. section 2.4. The book [5] is a good general reference on Einstein metrics. In particular, [5, chapter 12] contains a discussion of the moduli space of Einstein structures. In [5], the space of Einstein geometries on a compact manifold $M$ is studied as the space of metrics of unit volume, modulo diffeomorphisms, which solve the equation

$$
\mathrm{Ric}=\frac{1}{n}\left(\int_{M} \mu_{g} \mathrm{Scal}\right) g .
$$

The scalar curvature is locally constant on the space of Einstein geometries (cf. [5, corollary 12.52]). In this paper, we are interested only in connected components of the space of Einstein geometries with fixed negative Einstein constant. Thus, we fix $\alpha<0$ and consider without loss of generality the space $\mathcal{E}_{\alpha}$ of Einstein metrics on $M$ with Einstein constant $\alpha$, i.e. the space of solutions to the Riemannian Einstein equation

$$
\operatorname{Ric}=\alpha g
$$


We assume that $\mathcal{E}_{\alpha}$ is non-empty. The results from [5] specialize to the situation considered here. As in [5], we shall work with the premoduli space $\mathcal{E}_{\alpha} \cap \mathcal{S}$, where $\mathcal{S}$ is a slice for the diffeomorphism group. Given a metric $\gamma_{0} \in \mathcal{E}_{\alpha}$, we refer to the connected component of the premoduli space of $\gamma_{0}$ as the deformation space of $\gamma_{0}$. The moduli space of $\gamma_{0}$ is the quotient of the premoduli space by the isometry group of $\left(M, \gamma_{0}\right)$, which in case $\alpha<0$ is finite; see $[\mathbf{5}, \S 12 . \mathrm{C}]$ for further discussion.

2.1. The linearized Einstein equation. Denote by $\mathcal{A}$ the operator

$$
\mathcal{A} h=2 D(\text { Ric }-\alpha g) h,
$$

i.e. twice the Frechet derivative of $g \mapsto$ Ric $-\alpha g$, in the direction $h$, evaluated at $\gamma \in \mathcal{E}_{\alpha}$. Let $\mathcal{L}$ be given by

$$
\mathcal{L} h=-\Delta h-2 \stackrel{\circ}{R} h
$$

where $\Delta=\nabla^{a} \nabla_{a}$ and

$$
\stackrel{\circ}{R} h_{a b}=R_{a c b d} h^{c d} .
$$

Then

$$
\mathcal{A} h=\mathcal{L} h-2 \delta^{*} \delta h-\nabla d(\operatorname{tr} h)
$$

where

$$
\delta h_{a}=\nabla^{b} h_{a b}, \quad \delta^{*} \xi_{a b}=-\frac{1}{2}\left(\nabla_{a} \xi_{b}+\nabla_{b} \xi_{a}\right) .
$$

Symmetric two-tensors with vanishing trace and divergence, i.e. elements of ker $\delta \cap$ ker tr, play an important role in analyzing the linearized Einstein equation. Such tensors are called transverse and traceless or TT tensors. The space

$$
\operatorname{ker} \mathcal{A} \cap \operatorname{ker} \delta \cap \operatorname{ker} \operatorname{tr}
$$

is the space of infinitesimal Einstein deformations in our setting; cf. [5, theorem 12.30] for the analogous statement in their setting.

The operator $\mathcal{L}$ is a self-adjoint elliptic operator, and since by assumption $M$ is compact, $\mathcal{L}$ has discrete spectrum and finite dimensional kernel.

We remark that $\mathcal{L}$ is closely related to the Lichnerowicz Laplacian $\Delta_{L}$ defined by

$$
\Delta_{L} h=\mathcal{L} h+2 \operatorname{Ric} \circ h
$$

where for two symmetric tensors $u, v$,

$$
(u \circ v)_{a b}=\frac{1}{2}\left(u_{a c} v_{b}^{c}+v_{a c} u_{b}^{c}\right) .
$$

Let $\Delta_{H}=d \delta+\delta d$ be the Hodge Laplacian, where $\delta$ is the adjoint of $d$. For $\gamma \in \mathcal{E}_{\alpha}$ the commutation formulas

$$
\delta \mathcal{L} u=\left(\Delta_{H}-2 \alpha\right) \delta u
$$


and

$$
\operatorname{tr} \mathcal{L} u=(-\Delta-2 \alpha) \operatorname{tr} u
$$

hold. The Hodge Laplacian acts on one-forms by

$$
\Delta_{H} \xi=(-\Delta+\text { Ric }) \xi \text {. }
$$

In particular, $\Delta_{H}$ has nonnegative spectrum. It follows from the commutation formulas that $\mathcal{L}$ maps TT tensors to TT tensors. Further, if $\alpha<0$, then $\operatorname{ker} \mathcal{L} \subset \operatorname{ker} \delta \cap \operatorname{ker} \operatorname{tr}$, i.e. a subspace of the space of TT tensors.

For a symmetric 2-tensor $h$, we have the decomposition

$$
h=f g+h^{\mathrm{TT}}+\mathcal{L}_{Y} g,
$$

valid at any metric $g$, where $f$ is a function, $h^{\mathrm{TT}}$ is a TT tensor with respect to $g$, and $Y$ is a vector field. The equation Ric $=\alpha g$ is covariant, which implies that at $\gamma \in \mathcal{E}_{\alpha}, \mathcal{A}\left(\mathcal{L}_{Y} \gamma\right)=0$, for any vector field $Y$. In the rest of this subsection, we evaluate $\mathcal{L}$ and $\mathcal{A}$ at $\gamma \in \mathcal{E}_{\alpha}$. Since $\mathcal{A} h^{\mathrm{TT}}=\mathcal{L} h^{\mathrm{TT}}$, a calculation shows

$$
\mathcal{A} h=\mathcal{L} h^{\mathrm{TT}}+[(-\Delta-2 \alpha) f] \gamma+(2-n) \nabla d f .
$$

Taking the trace, we find that if $\alpha<0, h \in \operatorname{ker} \mathcal{A}$ only if $f=0$. This shows

$$
\text { ker } \mathcal{A}=\operatorname{ker} \mathcal{L}+\left\{\mathcal{L}_{Y} \gamma, Y \text { vector field on } M\right\} .
$$

Let $\mathbb{P} \|$ denote the $L^{2}$-orthogonal projection in the space of symmetric 2tensors onto the finite dimensional kernel ker $\mathcal{L}$, and similarly let $\mathbb{P}^{\perp}$ be the $L^{2}$-orthogonal projection in the space of symmetric 2-tensors onto the $L^{2}$-orthogonal complement of $\operatorname{ker} \mathcal{L}$ in the space of TT tensors with respect to $\gamma$.

Given a symmetric 2-tensor $u$, we will often use the notation $u^{\mathrm{TT}}$, $u^{\mathrm{TT} \|}$ and $u^{\mathrm{TT}} \perp$ for the TT part of $u$, in the sense of the decomposition (2.4), and the projections $\mathbb{P}^{\|} u, \mathbb{P}^{\perp} u$, respectively. In particular, a TT tensor $u^{\mathrm{TT}}$ can be decomposed as $u^{\mathrm{TT}}=u^{\mathrm{TT} \|}+u^{\mathrm{TT} \perp}$. If it is not clear from the context, we will indicate the dependence on the metric by, e.g., $\mathbb{P}_{\gamma}^{\|}$. If ker $\mathcal{L}=\{0\}$, then $\mathbb{P}^{\perp}$ is the projection onto the space of $\mathrm{TT}$ tensors with respect to $\gamma$.

2.2. Harmonic coordinates and the slice. For $\gamma \in \mathcal{E}_{\alpha}$, a slice $\mathcal{S}_{\gamma}$ for the diffeomorphism group, called the harmonic slice through $\gamma$, can be defined as follows. Let $\mathcal{S}_{\gamma}$ be the set of $g \in \mathcal{M}$ such that the identity map i : $(M, g) \rightarrow(M, \gamma)$ is harmonic. This condition holds if and only if the tension field $V$ vanishes, where $V$ is given by

$$
V^{i}[g ; \gamma]=g^{m n}\left(\Gamma[g]_{m n}^{i}-\Gamma[\gamma]_{m n}^{i}\right)
$$

For $\gamma \in \mathcal{E}_{\alpha}$ for $\alpha<0$ and $g$ sufficiently close to $\gamma$, we have that

$$
g^{m n} R[\gamma]_{i m j n} X^{i} X^{j} \leq-\lambda^{2}|X|_{\gamma}^{2}
$$


for some $\lambda>0$. In fact, it is sufficient for $\gamma$ to have negative Ricci curvature for this to hold. Therefore we have, following $[\mathbf{2}, \S 5]$ (see in particular $[\mathbf{2},(5.7)])$ that the operator $P$ defined by

$$
P X=D V \cdot \mathcal{L}_{X} \gamma,
$$

where the Frechet derivative of $V$ is taken with respect to $g$, is an isomorphism at $\gamma$, and the same holds, by continuity, for the corresponding operator defined at $g$, for $g$ close to $\gamma$. Consider a symmetric 2-tensor $h$ decomposed as in (2.4), with respect to $g$. We have for $g \in \mathcal{S}_{\gamma}$,

$$
(D V . h)^{i}=P Y^{i}+\left(1-\frac{n}{2}\right) \nabla^{i} f-h^{\mathrm{TT} m n}\left(\Gamma[g]_{m n}^{i}-\Gamma[\gamma]_{m n}^{i}\right) .
$$

Based on this, it is not difficult to apply the implicit function theorem to show that $\mathcal{S}_{\gamma}$ is a submanifold of $\mathcal{M}$ near $\gamma$. It follows from the same analysis that $\mathcal{S}_{\gamma}$ defined in this way is a slice for the diffeomorphism group; see also $[\mathbf{5}, \S 12 . \mathrm{C}]$ for discussion.

On the other hand, for $g \in \mathcal{M}$, close to a negative Einstein metric $\gamma$, there is a harmonic map $\phi:(M, g) \rightarrow(M, \gamma)$, with $\phi \in \mathcal{D}$ being the unique solution to the harmonic map equation

$$
\Delta \phi^{i}+\Gamma[\gamma]_{m n}^{i} \partial_{k} \phi^{m} \partial_{l} \phi^{n} g^{k l}=0
$$

in a neighborhood of the identity map i. This is proved along the lines of the above remarks, also using an implicit function theorem argument. Given $\phi$, the pushforward $\left(\phi^{-1}\right)^{*} g$ has the property that i : $\left(M,\left(\phi^{-1}\right)^{*} g\right) \rightarrow(M, \gamma)$ is harmonic, and also that $\mathbf{i}:(M, g) \rightarrow\left(M, \phi^{*} \gamma\right)$ is harmonic. Since $\gamma \in \mathcal{E}_{\alpha}$, this holds for $\phi^{*} \gamma$ too. Thus, if $g$ is close in $\mathcal{M}$ to $\gamma \in \mathcal{E}_{\alpha}$, we may, after applying a diffeomorphism, assume that in fact $g \in \mathcal{S}_{\gamma}$. If it is clear from the context which metric is used to define the slice, we will simply denote the slice by $\mathcal{S}$.

2.3. The deformation space. Fix $\gamma_{0} \in \mathcal{E}_{\alpha}$ and for the rest of this section, let $\mathcal{S}$ be the slice defined with respect to $\gamma_{0}$.

Definition 2.1. Let $\gamma_{0} \in \mathcal{E}_{\alpha}$, and let $\mathcal{V}$ be the connected component of $\gamma_{0}$ in $\mathcal{E}_{\alpha}$. The space $\mathcal{N}=\mathcal{V} \cap \mathcal{S}$ is called the deformation space of $\gamma_{0}$. If $\mathcal{N}=\left\{\gamma_{0}\right\}$, then $\gamma_{0}$ is called rigid.

Remark 2.2. By [5, corollary 12.52], the moduli space of Einstein structures is locally connected. In the terminology of $[\mathbf{5}, \S 12]$, the deformation space is the connected component of the premoduli space, which contains $\gamma_{0}$.

By definition, $\mathcal{N}$ is the $\gamma_{0}$ component in the space of solutions of

$$
\text { Ric }=\alpha g, \quad V=0 .
$$

By (2.6), $h$ solves the linearization of the equation Ric $=\alpha g$ at $\gamma_{0}$ if and only if

$$
h=h^{\mathrm{TT} \|}+\mathcal{L}_{Y} \gamma_{0},
$$


with $h^{\mathrm{TT}} \| \in \operatorname{ker} \mathcal{L}$, where $\mathcal{L}$ is the operator defined by (2.1) at $\gamma_{0}$. From the discussion in section 2.2 , we see that the space of solutions to the linearization of the system $(2.10)$ at $\gamma_{0}$ is equivalent to $\operatorname{ker} \mathcal{L}$. By $[\boldsymbol{5}$, corollary 12.66], $\gamma_{0}$ is rigid if $\operatorname{ker} \mathcal{L}=0$.

Next we consider the linearization of the system (2.10) away from $\gamma_{0}$. Thus, let $\gamma \in \mathcal{N}$ be close to $\gamma_{0}$. Again, the space of solutions of the linearized Riemannian Einstein equations has the form (2.11). However, for $\gamma \neq \gamma_{0}$, the term

$$
h^{\mathrm{TT} \| m n}\left(\Gamma[\gamma]_{m n}^{i}-\Gamma\left[\gamma_{0}\right]_{m n}^{i}\right)
$$

is nontrivial, and the space of solutions of the linearization of the system (2.10) is of the form

$$
h^{\mathrm{TT} \|}+\mathcal{L}_{Y \|} \gamma,
$$

where $h^{\mathrm{TT}} \| \in \operatorname{ker} \mathcal{L}$ with $\mathcal{L}$ defined with respect to $\gamma$, and $Y^{\|}$solves the equation

$$
P Y^{\| i}-h^{\mathrm{TT} \| m n}\left(\Gamma[\gamma]_{m n}^{i}-\Gamma\left[\gamma_{0}\right]_{m n}^{i}\right)=0 .
$$

Here $P$ is the operator given by (2.8), defined with respect to $\gamma$ with background metric $\gamma_{0}$. Thus the space of $h$ of the form (2.12) is the formal tangent space of the deformation space $\mathcal{N}$ at $\gamma$.

Definition 2.3. If $\mathcal{N}$ is a manifold near $\gamma$, with tangent space given by the formal tangent space, i.e.

$$
T_{\gamma} \mathcal{N}=\left\{h: h=h^{\mathrm{TT}} \|+\mathcal{L}_{Y \|} \gamma\right\},
$$

with $Y^{\|}$a solution to (2.13), then $\mathcal{N}$ is called integrable at $\gamma$.

See $[\mathbf{5}, \S \S 12 . \mathrm{E}, \mathrm{F}]$ for a discussion of integrability.

Remark 2.4. No example of a compact, negative Einstein space with non-integrable deformation space is known.

From now on, we shall consider only integrable deformation spaces.

\subsection{Stable negative Einstein spaces.}

Definition 2.5. Let $(M, \gamma)$ be a negative Einstein space and let $\lambda_{\min }$ be the lowest eigenvalue of $\mathcal{L}$. We call $(M, \gamma)$ stable if $\lambda_{\min } \geq 0$, while if $\lambda_{\min }>0,(M, \gamma)$ is called strictly stable.

Remark 2.6. No example of an unstable, compact, negative Einstein space is known.

The following lemma shows that stability can be analyzed by looking at the restriction of $\mathcal{L}$ to TT tensors.

Lemma 2.7. Suppose $(M, \gamma)$ is an Einstein space with Einstein constant $\alpha<0$. Then $\mathcal{L}$ has nonnegative spectrum as an operator on TT tensors if and only if $(M, \gamma)$ is stable. In particular, $\operatorname{ker} \mathcal{L} \subset \operatorname{ker} \delta \cap \operatorname{ker} \operatorname{tr}$ so any element of $\operatorname{ker} \mathcal{L}$ is a TT tensor. 
Proof. Let $u$ be an eigentensor of $\mathcal{L}$,

$$
\mathcal{L} u=\lambda u
$$

and suppose $\lambda \leq 0$. This implies

$$
\delta \mathcal{L} u=\lambda \delta u
$$

and hence

$$
\Delta_{H} \delta u=(2 \alpha+\lambda) \delta u,
$$

i.e. either $2 \alpha+\lambda<0$ is an eigenvalue of $\Delta_{H}$ or $\delta u=0$. Since $\Delta_{H}$ is nonnegative, we have $\delta u=0$. Similarly, by applying the trace to both sides, we find

$$
-\Delta \operatorname{tr} u=(2 \alpha+\lambda) \operatorname{tr} u,
$$

which implies $\operatorname{tr} u=0$. Hence $u$ is a TT tensor. This shows that if $\mathcal{L}$ has nonnegative spectrum when restricted to TT tensors, then $\mathcal{L}$ has nonnegative spectrum. The converse is immediate, as is the statement about ker $\mathcal{L}$. q.e.d.

Proposition 2.8. 1) If $\gamma_{0} \in \mathcal{E}_{\alpha}$ is strictly stable, then $\mathcal{N}=\left\{\gamma_{0}\right\}$. $\gamma_{0}$ is isolated in $\mathcal{E}_{\alpha} \cap \mathcal{S}$. In particular, there is a neighborhood $\mathcal{U}$ of $\gamma_{0}$ in the space of metrics such that $\mathcal{U} \cap \mathcal{E}_{\alpha} \cap \mathcal{S}=\left\{\gamma_{0}\right\}$.

2) If $\gamma_{0} \in \mathcal{E}_{\alpha}$ has integrable, nontrivial deformation space, then there is a neighborhood $\mathcal{U}$ of $\gamma_{0}$ in the space of metrics, such that $\mathcal{U} \cap$ $\mathcal{E}_{\alpha} \cap \mathcal{S}=\mathcal{U} \cap \mathcal{N}$.

Proof. Point 1 is a special case of [5, corollary 12.66]. Point 2 is the fact that the moduli space and in particular the premoduli space is locally arcwise connected; cf. [5, corollary 12.52].

q.e.d. 2.5. Examples. We will now discuss some general conditions which imply that a negative Einstein space is stable or strictly stable, and give examples of such spaces as well as of stable negative Einstein spaces with nontrivial deformation space.

2.5.1. Strictly stable spaces. A compact Einstein space of dimension $\geq 3$, with negative sectional curvature, is strictly stable; cf. $[\mathbf{5}, \S 12$ $\mathrm{H}]$. Thus, in particular, compact rank one symmetric spaces of noncompact type provide examples of strictly stable negative Einstein spaces. These include, among others, compact hyperbolic and complex hyperbolic spaces. More generally, locally symmetric spaces of noncompact type, which have no local 2-dimensional factor, are rigid [5, prop. 12.74] and hence also strictly stable. We mention also the work of Fischer and Moncrief [10], which gives a condition on the Weyl tensor that implies strict stability.

Remark 2.9. In certain cases, a type of uniformization theorem is valid. If $M$ carries a hyperbolic metric, then by work of Besson et al. [6], an Einstein metric on $M$ is hyperbolic. Further, in dimension 4, if $M$ carries a complex hyperbolic metric, then any Einstein metric on $M$ is complex hyperbolic by work of LeBrun [12]. 
2.5.2. Nontrivial deformation spaces. In the two dimensional case, negative curvature does not imply strict stability. In particular, from standard results in Teichmüller theory, in the case $n=2$, a Riemann surface $M$ of $\operatorname{genus}(M)>1$ with the hyperbolic metric is a stable negative Einstein space with nontrivial, integrable deformation space, namely the Teichmüller space. In this case, $\operatorname{dim} \operatorname{ker} \mathcal{L}=6 \operatorname{genus}(M)-6$, the dimension of the Teichmüller space of $M$.

Higher dimensional examples of negative Einstein spaces with nontrivial integrable deformation spaces are provided by Kähler-Einstein metrics. By a result of Aubin and Yau, cf. [5, theorem 11.17], any compact complex manifold with negative first Chern class admits a unique Kähler-Einstein metric $(\gamma, J)$, where $J$ is the complex structure, with Einstein constant $\alpha<0$. A result by Koiso [5, theorem 12.88] shows that the deformation space of $\gamma$ is integrable if all infinitesimal complex deformations of $J$ are integrable. Examples where these conditions hold are provided by hypersurfaces of $\mathbb{C} P^{m}, m \geq 3$, of degree $d \geq m+2$; cf. [5, example 12.89].

By a theorem of Dai et al. [8], compact Kähler-Einstein spaces are stable. The proof uses the fact that Kähler-Einstein spaces carry parallel $\operatorname{spin}^{c}$ spinors. In the presence of a parallel spinor $\sigma_{0}$, it is possible to relate $\mathcal{L}$ to the square of the Dirac operator. Let $\Sigma(M)$ denote the $\operatorname{spin}^{c}$ spinor bundle of $M$. Define the map $\Phi: S^{2} T^{*} M \rightarrow \Sigma(M) \otimes T^{*} M$, taking symmetric 2-tensors to spinor-valued 1-forms, by

$$
\Phi(h)=h_{j}^{i} e_{i} \sigma_{0} \otimes e^{j}
$$

where $\left(e_{i}\right)$ is an ON frame on $(M, \gamma)$ with dual frame $\left(e^{i}\right)$, and $h_{i j}=$ $h\left(e_{i}, e_{j}\right)$ are the frame components of $h$. Here indices are raised using $\gamma^{i j}$, which is just $\delta^{i j}$. A calculation shows

$$
D^{*} D \Phi(h)=\Phi(\mathcal{L} h-h \circ F+\operatorname{Ric} \circ h)
$$

where $D$ is the Dirac operator, $F$ is the curvature of the line bundle appearing in the $\operatorname{spin}^{c}$ structure, and $(h \circ F)_{a b}=h_{a}{ }^{c} F_{c b}$, and (Ric $\circ$ $h)_{a b}=\operatorname{Ric}_{a c} h_{b}^{c}$. The terms involving $F$, Ric give a positive semi-definite contribution, and one can show

$$
(\mathcal{L} h, h)_{L^{2}} \geq(D \Phi(h), D \Phi(h))_{L^{2}} \geq 0,
$$

for all $h$; cf. [8, theorem 2.4].

\section{Stability of Product Spaces}

3.1. Tensors on product spaces. Let $M, N$ be compact, connected, stable negative Einstein manifolds with constant $\alpha<0$, of dimension $m, n$ and with metrics $\gamma^{M}, \gamma^{N}$ respectively. Then $M \times N$ is an Einstein space with constant $\alpha$ and metric $\gamma=\left(\pi^{M}\right)^{*} \gamma^{M}+\left(\pi^{N}\right)^{*} \gamma^{N}$, where $\pi^{M}, \pi^{N}$ denote the projections of $M \times N$ to the factors $M, N$, respectively. In the following we shall lift tensors on the factors $M, N$ to 
tensors on the product $M \times N$ by pulling back along the projections $\pi^{M}, \pi^{N}$, and in order to avoid notational complications, for the rest of this section we drop explicit reference to the projections. Thus, for example, we write the metric on $M \times N$ simply as $\gamma^{M}+\gamma^{N}$.

For tensors on $M$, we use Greek indices $\mu, \nu, \gamma, \ldots$, and for tensors on $N$, we use uppercase Latin indices $A, B, C, \ldots$ For tensors on the product $M \times N$, we use lowercase Latin indices $a, b, c, \ldots$, or, when convenient, a mixture of the two other index types. For computations involving tensor products, we will use both index and index-free notation. We will write $\nabla^{M}, \nabla^{N}$ for the $M, N$ covariant derivatives, respectively, and $\nabla$ for the $M \times N$ covariant derivative. Similarly, $\Delta^{M}, \Delta^{N}$ denote the Laplacians on $M, N$ and $\Delta$ the Laplacian on $M \times N$. The space of symmetric covariant tensors of order $k$ on $M$ will be denoted by $\mathcal{S}^{k} M$, and the space of covariant $k$-tensors on $M$ will be denoted by $\mathcal{T}^{k} M$, and similarly for the other spaces. The covariant derivatives on $M, N$ apply in an unambiguous way to lifted tensors. For example, let $\xi, \eta$ be tensors on $M, N$. Then with the above notation we have

$$
\nabla(\xi \otimes \eta)=\left(\nabla^{M} \xi\right) \otimes \eta+\xi \otimes\left(\nabla^{N} \eta\right)
$$

as well as the obvious index version of this formula. Similarly, we have

$$
\Delta(\xi \otimes \eta)=\left(\Delta^{M} \xi\right) \otimes \eta+\xi \otimes\left(\Delta^{N} \eta\right)
$$

in particular, there are no cross terms. Let $\odot$ be the symmetric tensor product, by definition

$$
\xi \odot \eta=\xi \otimes \eta+\eta \otimes \xi .
$$

Then

$$
\nabla(\xi \odot \eta)=\left(\nabla^{M} \xi\right) \odot \eta+\xi \odot\left(\nabla^{N} \eta\right)
$$

and analogously for $\Delta$.

We will frequently consider symmetric tensors on $M \times N$ of the form

$$
t=u \psi+\xi \odot \eta+\phi v
$$

where $u \in \mathcal{S}^{2}(M), \xi \in \mathcal{T}^{1}(M), \phi \in C^{\infty}(M), \psi \in C^{\infty}(N), \eta \in \mathcal{T}^{1}(N)$, $v \in \mathcal{S}^{2}(N)$. Then

$$
\begin{aligned}
\nabla t=\nabla^{M} u \psi+u \otimes \nabla^{N} \psi+\left(\nabla^{M} \xi\right) & \odot \eta \\
& +\xi \odot\left(\nabla^{N} \eta\right)+\nabla^{M} \phi \otimes v+\phi \nabla^{N} v
\end{aligned}
$$

and similarly for $\Delta$. For a Cartesian product $M \times N$, the cross terms in the Riemann tensor vanish in the sense that $R(X, Y, Z, W)=0$ whenever $X, Y, Z, W$ contains a pair of vector fields which are tangent to $M, N$ respectively. It follows that the operators $\stackrel{\circ}{R}$ defined in terms of the Riemann tensor as in (2.2) act block diagonally on tensor products formed of tensors on $M$ and $N$. In particular, with $\xi, \eta$ as above,

$$
\stackrel{\circ}{R}(\xi \odot \eta)=0 .
$$


Hence, we have

$$
\begin{aligned}
\mathcal{L} t= & -u \Delta^{N} \psi-\psi \Delta^{M} u-\Delta^{M} \xi \odot \eta-\xi \odot \Delta^{N} \eta \\
& -v \Delta^{M} \phi-\phi \Delta^{N} v-2 \psi\left(\stackrel{\circ}{R}_{M} u\right)-2 \phi\left(\stackrel{\circ}{R}_{N} v\right)
\end{aligned}
$$

where $\stackrel{\circ}{R}_{M}, \stackrel{\circ}{R}_{N}$ are defined in terms of the Riemann tensors $R_{M}, R_{N}$ of $M, N$, respectively.

3.2. Spectral decomposition. Consider the compact, negative Einstein spaces $M, N$ as above. Since we are considering compact manifolds, the covariant Laplacian acting on functions and one-forms and $\mathcal{L}$ acting on symmetric 2-tensors are self-adjoint, with discrete spectrum. We will use the following notation for the spectral decompositions. On $M$ the eigenvalues for the operators $-\Delta,-\Delta, \mathcal{L}$ acting on tensors of order $k=0,1,2$ will be denoted by $\lambda_{i}^{(k)}$. The $L^{2}$ normalized eigentensors of order $0,1,2$ will be denoted by $\phi_{i}, \xi_{i}, u_{i}$, respectively. Similarly, on $N$, the eigenvalues will be denoted $\mu_{i}^{(k)}, k=0,1,2$, and the $L^{2}$ normalized eigentensors of order $0,1,2$ will be denoted by $\psi_{i}, \eta_{i}, v_{i}$. Then from spectral theory, it follows that the $\left\{\phi_{i}\right\},\left\{\xi_{i}\right\},\left\{u_{i}\right\}$ and $\left\{\psi_{i}\right\},\left\{\eta_{i}\right\},\left\{v_{i}\right\}$ constitute $L^{2}$ bases for tensors of order $0,1,2$ on $M$ and $N$, respectively.

Lemma 3.1. The tensor products $u_{i} \psi_{j}, \xi_{i} \odot \eta_{j}, \phi_{i} v_{j}$ form a complete orthonormal system in $\mathcal{S}^{2}(M \times N)$.

Proof. It is clear that $\left\{u_{i} \psi_{j}\right\},\left\{\xi_{i} \odot \eta_{j}\right\}$, and $\left\{\phi_{i} v_{j}\right\}$ are orthonormal sets. Let $f \in \mathcal{S}^{2}(M \times N)$. We can assume without loss of generality that $f$ is smooth. Let $x^{\mu}$ and $y^{A}$ be coordinate systems on $M, N$ so that $\left(x^{\mu}, y^{A}\right)$ is a coordinate system on $M \times N$. Then we can write $f=s_{\mu \nu} d x^{\mu} \odot d x^{\nu}+t_{\mu A} d x^{\mu} \odot d y^{A}+u_{A B} d y^{A} \odot d y^{B}$, where the coefficients are smooth functions of $x, y$. Now suppose $f$ is $L^{2}$ perpendicular to all $u_{i} \psi_{j}$. This implies for all $i, j$,

$$
\int_{N}\left(\int_{M}\left\langle s(x, y), u_{i}(x)\right\rangle \mu_{M}(x)\right) \psi_{j} \mu_{N}(y)=0 .
$$

Since $\left\{\psi_{j}\right\}$ is a basis for $L^{2}(N)$,

$$
\int_{M}\left\langle s(x, y), u_{i}(x)\right\rangle \mu_{M}(x)=0,
$$

from which follows $s=0$. We can deal with the other factors similarly.

q.e.d.

Lemma 3.2. Let $M, N$ be stable Einstein spaces with Einstein constant $\alpha<0$. Then $M \times N$ is stable and

$$
\operatorname{ker} \mathcal{L}^{M \times N}=\operatorname{ker} \mathcal{L}^{M}+\operatorname{ker} \mathcal{L}^{N} .
$$


Proof. We will drop the superscript on $\mathcal{L}$ and other operators when it is clear from the context which space they act on. By Lemma 3.1, any tensor $t \in \mathcal{S}^{2}(M \times N)$ can be expanded in the form

$$
t=\sum_{i, j} a_{i j} u_{i} \psi_{j}+\sum_{i, j} b_{i j} \xi_{i} \odot \eta_{j}+\sum_{i, j} c_{i j} \phi_{i} v_{j}
$$

with constants $\left(a_{i j}, b_{i j}, c_{i j}\right)$. A calculation shows

$$
\begin{aligned}
\mathcal{L} t & =\sum_{i, j} a_{i j}\left(\lambda_{i}^{(2)}+\mu_{j}^{(0)}\right) u_{i} \psi_{j} \\
& +\sum_{i, j} b_{i j}\left(\lambda_{i}^{(1)}+\mu_{j}^{(1)}\right) \xi_{i} \odot \eta_{j} \\
& +\sum_{i j} c_{i j}\left(\lambda_{i}^{(0)}+\mu_{j}^{(2)}\right) \phi_{i} v_{j} .
\end{aligned}
$$

Thus eigentensors of the operator $\mathcal{L}$ on $M \times N$ can be of order $(2,0)$, $(1,1)$, and $(0,2)$, with eigenvalues $\lambda_{i}^{(2)}+\mu_{j}^{(0)}, \lambda_{i}^{(1)}+\mu_{j}^{(1)}$, and $\lambda_{i}^{(0)}+\mu_{j}^{(2)}$, respectively. Now assume each factor $M, N$ is stable, i.e. the operators $\mathcal{L}^{M}, \mathcal{L}^{N}$ are nonnegative when acting on TT tensors. By Lemma 2.7, this implies $\mathcal{L}^{M}, \mathcal{L}^{N}$ are nonnegative when acting on all tensors on $M, N$ respectively. Further, the operator $-\Delta$ acting on functions is nonnegative, and since $\Delta_{H}=-\Delta+$ Ric is nonnegative, it follows that $-\Delta$ acting on 1 -forms has spectrum bounded from below by $-\alpha>0$. Hence, the operator $\mathcal{L}$ on $M \times N$ is nonnegative.

It remains to identify the kernel of $\mathcal{L}$. Suppose $\mathcal{L} t=0$. Recall that $\mathcal{L}$ acts on the off diagonal terms by $-\Delta$ which has spectrum bounded from below by $-\alpha>0$. Hence $t$ must have coefficients $b_{i j}=0$. Examining the action of $\mathcal{L}$ on $t$, we see that zero in the spectrum of $\mathcal{L}$ corresponds to zero in the spectrum of $\mathcal{L}^{M}, \mathcal{L}^{N}$ as well as zero in the spectrum of $\Delta^{M}, \Delta^{N}$. Since the zero eigenfunction of the scalar Laplacian is constant, we see that $t=u+v$, with $u \in \operatorname{ker} \mathcal{L}^{M}$ and $v \in \operatorname{ker} \mathcal{L}^{N}$. This completes the proof of the theorem.

q.e.d.

The result of Lemma 3.2 clearly applies to an arbitrary number of factors.

3.3. Deformation spaces on products. In the following we will consider products with two factors. However, as in the case of Lemma 3.2, the results in the rest of this section apply to products with an arbitrary number of factors.

We assume the deformation spaces $\mathcal{N}^{M}, \mathcal{N}^{N}$ of the background spaces $\left(M, \gamma_{0}^{M}\right),\left(N, \gamma_{0}^{N}\right)$ are integrable and stable. We allow the case where one or both of $\gamma_{0}^{M}, \gamma_{0}^{N}$ are strictly stable so that the corresponding deformation space is trivial. 
Proposition 3.3. Let $\left(M, \gamma_{0}^{M}\right),\left(N, \gamma_{0}^{N}\right)$ be stable Einstein spaces with Einstein constant $\alpha$. Assume that $M, N$ have integrable deformation spaces. Then, the deformation space $\mathcal{N}$ of $\gamma$ is locally diffeomorphic to $\mathcal{N}^{M} \times \mathcal{N}^{N}$.

Proof. Clearly $\mathcal{N}$ contains the space $\mathcal{N}^{M} \times \mathcal{N}^{N}$. Let $\mathcal{S}$ be the slice for $M \times N$ defined with respect to the product metric $\gamma_{0}=\gamma_{0}^{M}+\gamma_{0}^{N}$ and let $\mathcal{S}^{M}, \mathcal{S}^{N}$ be the slices defined with respect to $\gamma_{0}^{M}, \gamma_{0}^{N}$. By Lemma 2.7 and Lemma 3.2, we have $T_{\gamma_{0}}\left[\left(\mathcal{V}^{M} \times \mathcal{V}^{N}\right) \cap \mathcal{S}\right]=\operatorname{ker} \mathcal{L}=$ $\operatorname{ker} \mathcal{L}^{M}+\operatorname{ker} \mathcal{L}^{N}=T_{\gamma_{0}^{M}}\left(\mathcal{V}^{M} \cap \mathcal{S}^{M}\right)+T_{\gamma_{0}^{N}}\left(\mathcal{V}^{N} \cap \mathcal{S}^{N}\right)$. Therefore, the tangent space of the deformation space at $\gamma_{0}$ is equal to the formal tangent space. It follows from $[\mathbf{5} \text {, theorem } 13.49, \text { p. } 351]^{1}$ that the deformation space is integrable near $\gamma_{0}$.

q.e.d.

The following is an immediate corollary to Propositions 3.3 and 2.8.

Corollary 3.4. There is a neighborhood $\mathcal{U}$ of $\gamma_{0}$ in $\mathcal{M}(M \times N)$ such that $\mathcal{U} \cap \mathcal{E}_{\alpha}(M \times N) \cap \mathcal{S}=\mathcal{U} \cap\left(\mathcal{N}^{M} \times \mathcal{N}^{N}\right)$.

3.4. Examples of stable product spaces. The results above show that Cartesian products of spaces which are strictly stable, or stable and integrable, yield spaces which are stable and integrable. If all factors are strictly stable, then the product is strictly stable. Thus the examples discussed in section 2.5 allow us to construct large families of stable integrable negative Einstein spaces with nontrivial deformation spaces, as well as large families of strictly stable negative Einstein spaces.

Among the cases of interest are products of hyperbolic manifolds with hyperbolic surfaces, as well as products of hyperbolic manifolds with negative Kähler-Einstein spaces.

\section{The Einstein Evolution Equations}

Let $\gamma \in \mathcal{E}_{-(n-1) / n^{2}}$. Then the Lorentz cone over $(M, \gamma)$, i.e. the manifold $(0, \infty) \times M$ with metric

$$
-d t \otimes d t+\frac{t^{2}}{n^{2}} \gamma
$$

is a Ricci flat, maximal, globally hyperbolic spacetime, which admits a timelike homothety $t \partial_{t}$.

In this section we write the Einstein evolution equations in terms of scale invariant variables. As we shall see, the resulting system is autonomous, and data corresponding to the Lorentz cone metric (4.1) is an equilibrium point for this system.

\footnotetext{
${ }^{1}$ Due to a typographical error in [5], theorem 12.49 appears as theorem 13.49 .
} 
4.1. Scale invariant variables. Let $(\tilde{g}, \tilde{K}, \tilde{N}, \tilde{X})$ be constant mean curvature Cauchy data for the Einstein equations. We use the same conventions as in [3]. Let $\tau=\operatorname{tr}_{\tilde{g}} \tilde{K}$ denote the mean curvature. We assume $\tau<0$. The rescaled variables corresponding to $(\tilde{g}, \tilde{K}, \tilde{N}, \tilde{X})$ are $\left(g_{i j}, \Sigma_{i j}, N, X^{i}\right)$, defined by

$$
\begin{array}{ll}
g_{i j}=\tau^{2} \tilde{g}_{i j}, & N=\tau^{2} \tilde{N}, \quad X^{i}=\tau \tilde{X}^{i}, \\
g^{i j}=\frac{1}{\tau^{2}} \tilde{g}^{i j}, & \Sigma_{i j}=\tau\left(\tilde{K}_{i j}-\frac{\tau}{n} \tilde{g}_{i j}\right) .
\end{array}
$$

Since we are assuming constant mean curvature, $\Sigma$ and $\tau$ contain the same information as $\tilde{K}$. Thus, it is natural to use $(g, \Sigma, N, X)$ as the set of scale invariant Cauchy data. In particular, for the line element (4.1), we have

$$
\tilde{g}(t)=\left(t^{2} / n^{2}\right) \gamma, \quad \tilde{K}(t)=-t^{-1} \tilde{g}, \quad \tau(t)=-n t^{-1}
$$

and hence the rescaled data are

$$
(g, \Sigma) \equiv(\gamma, 0)
$$

Note that by construction $\Sigma$ has vanishing trace. Thus, we view $(g, \Sigma)$ as an element of $T^{\operatorname{tr}} \mathcal{M}$, where $T^{\operatorname{tr}} \mathcal{M}$ denotes the subbundle of $T \mathcal{M}$ with fiber at $g$ consisting of symmetric tensors $h$ such that $\operatorname{tr}_{g} h=0$.

Introduce the scale invariant time $T$ by

$$
T=-\ln \left(\tau / \tau_{0}\right)
$$

where $\tau_{0}$ is some negative constant. Then, $\partial_{T}=-\tau \partial_{\tau}$. As we shall see below, the Einstein evolution equations in terms of the scale invariant variables, and the scale invariant time $T$, form an autonomous system.

4.2. Constraint set and slice. In this section, we review some results from $[3, \S 2.3]$ concerning the geometry of the constraint set and the slice for the diffeomorphism group determined by the spatial harmonic gauge condition. In $[\mathbf{3}, \S 2.3], M$ was assumed to have constant negative curvature. However, the results which we shall need generalize immediately to the case being considered here of negative Einstein spaces. In this paper we use the scale invariant metric and the shear tensor $(g, \Sigma)$ as fundamental variables, so we shall use these in the discussion of the constraint set and slice.

Let $\mathcal{C}$ be the space of $(g, \Sigma) \in T^{\operatorname{tr}} \mathcal{M}$ which are solutions to the vacuum constraint equations. Recalling that we are considering the constant mean curvature case, the constraint equations written in terms of the rescaled variables $(g, \Sigma)$ are of the form

$$
\begin{aligned}
& 0=R+\frac{n-1}{n}-|\Sigma|^{2}, \\
& 0=\nabla^{i} \Sigma_{i j},
\end{aligned}
$$


where $|\Sigma|^{2}=\Sigma_{i j} \Sigma^{i j}$. Fix a background metric $\gamma_{0} \in \mathcal{E}_{\alpha}$, let $\mathcal{N}$ be the deformation space with respect to $\gamma_{0}$, and let $\gamma \in \mathcal{N}$ be close to $\gamma_{0}$. By the discussion in section 2.2, there is a harmonic slice $\mathcal{S}_{\gamma} \subset \mathcal{M}$ defined with respect to $\gamma$. Corresponding to $\mathcal{S}_{\gamma}$, we have the slice $\mathcal{S}_{\mathcal{C}, \gamma}$ in the constraint set, ${ }^{2}$ consisting of solutions $(g, \Sigma)$ to the constraint equations such that $g \in \mathcal{S}_{\gamma}$. By [3, lemma 2.3], which generalizes immediately to the present situation, $\mathcal{S}_{\mathcal{C}, \gamma}$ is a smooth submanifold of $T^{\operatorname{tr}} \mathcal{M}$ with tangent space at $(\gamma, 0)$ given by the affine subspace

$$
\begin{aligned}
T_{(\gamma, 0)} \mathcal{S}_{\mathcal{C}, \gamma} & =(\gamma, 0) \\
+ & \left\{\left(u^{\mathrm{TT}}, v^{\mathrm{TT}}\right), \text { where } u^{\mathrm{TT}}, v^{\mathrm{TT}} \text { are TT-tensors w.r.t. } \gamma\right\}
\end{aligned}
$$

of $T_{\gamma}^{\mathrm{tr}} \mathcal{M}$. Given $\gamma$, we have the decomposition

$$
t=t^{\mathrm{TT}}+\left[\phi \gamma+\mathcal{L}_{X} \gamma\right]
$$

for any symmetric 2-tensor $t$, where $t^{\mathrm{TT}}$ is a TT tensor on $M$ with respect to $\gamma$, and $\phi, X$ are a function and a vector field on $M$, respectively. It is important to note that the terms $t^{\mathrm{TT}}$ and $\phi \gamma+\mathcal{L}_{X} \gamma$ are $L^{2}$-orthogonal. We can represent $\mathcal{S}_{\mathcal{C}, \gamma}$ as a graph over its tangent space. In particular, we may write $(g, \Sigma) \in \mathcal{S}_{\mathcal{C}, \gamma}$ in the form

$$
g=\gamma+u^{\mathrm{TT}}+z, \quad \Sigma=v^{\mathrm{TT}}+w
$$

with $z, w L^{2}$-orthogonal to the space of TT tensors defined with respect to $\gamma$. Then $(z, w)$ are second order in $\left(u^{\mathrm{TT}}, v^{\mathrm{TT}}\right)$, i.e. an estimate of the form

$$
\|z\|_{H^{s}}+\|w\|_{H^{s-1}} \leq C\left(\left\|u^{\mathrm{TT}}\right\|_{H^{s}}^{2}+\left\|v^{\mathrm{TT}}\right\|_{H^{s-1}}^{2}\right)
$$

holds.

4.3. Gauge condition and the shadow metric. We introduce the following modification of the CMCSH gauge of $[\mathbf{2}]$. Let $\mathcal{N}$ be the deformation space of $\gamma_{0}$ and assume that $\mathcal{N}$ is integrable.

Introducing a system of local coordinates $\left(q^{\alpha}\right), \alpha=1, \ldots, m=\operatorname{dim} \mathcal{N}$ on $\mathcal{N}$, we may write a general element of $\mathcal{N}$ as $\gamma_{i j}=\gamma_{i j}\left(q^{\alpha}\right)$. Then

$$
\frac{\partial \gamma_{i j}}{\partial q^{\alpha}}=h_{i j}^{(\alpha)}
$$

gives a basis for $T_{\gamma} \mathcal{N}$. From equation (2.12), we have that each $h_{i j}^{(\alpha)}$ admits a decomposition

$$
h_{i j}^{(\alpha)}=h_{i j}^{(\alpha) \mathrm{TT} \|}+\mathcal{L}_{Y^{(\alpha)} \|} \gamma_{i j}
$$

with $h_{i j}^{(\alpha) \mathrm{TT} \|} \in \operatorname{ker} \mathcal{L}_{\gamma}$, where $\mathcal{L}_{\gamma}$ is defined by (2.1) in terms of $\gamma$. In general, the term $\mathcal{L}_{Y^{(\alpha)} \|} \gamma_{i j}$, where $Y^{(\alpha)} \|$ is determined in terms of $h^{(\alpha) \mathrm{TT} \|}$ by equation $(2.13)$, is non-vanishing.

\footnotetext{
${ }^{2} \operatorname{In}[\mathbf{3}], \mathcal{S}_{\mathcal{C}}$ was denoted by $\Sigma$.
} 
For $g \in \mathcal{M}, \gamma \in \mathcal{N}$, we say that $g-\gamma$ is $L^{2}$-perpendicular to $\mathcal{N}$ at $\gamma$ if the conditions

$$
0=\left(g-\gamma, h^{(\alpha)}\right)_{L^{2} ; \gamma}, \quad \alpha=1, \ldots, m
$$

or explicitly,

$$
0=\int_{M}\left(g_{i j}-\gamma_{i j}\right) \frac{\partial \gamma^{i j}}{\partial q^{\alpha}} \mu_{\gamma}, \quad \alpha=1, \ldots, m
$$

hold. Here

$$
\frac{\partial \gamma^{i j}}{\partial q^{\alpha}}=-\left(\frac{\partial \gamma_{k l}}{\partial q^{\alpha}}\right) \gamma^{k i} \gamma^{l j}
$$

Remark 4.1. Equation (4.5) can be viewed as defining a smooth projection map $\mathcal{P}$ mapping a neighborhood of $\gamma_{0}$ in $\mathcal{M}$ to $\mathcal{N}$, given by $\mathcal{P}[g]=\gamma$. See section 4.5 for the relevant calculation. We refer to the map $\mathcal{P}$ as the shadow map. In view of the fact (cf. [5, chapter 5F]) that Einstein metrics are smooth (in fact, real analytic) in harmonic coordinates, it follows that $\mathcal{P}$ is smoothing in the sense that, for $g$ in the neighborhood of $\gamma_{0}$ where $\mathcal{P}$ is defined and regular, we have that $\left.D \mathcal{P}\right|_{g}: H^{s} \rightarrow H^{s^{\prime}}$ is continuous for any $s, s^{\prime}$.

Definition 4.2. Fix $\gamma_{0} \in \mathcal{E}_{-(n-1) / n^{2}}$, and let $\mathcal{N}$ be the deformation space defined with respect to $\gamma_{0}$; see section 2.3. Assume that $\mathcal{N}$ is integrable. We say that for $\gamma \in \mathcal{N}$, and Cauchy data $(g, \Sigma) \in \mathcal{C}$, the triple $(\gamma, g, \Sigma)$ satisfies the shadow metric condition if

1) $g$ satisfies the CMCSH gauge condition with respect to $\gamma$, i.e.

$$
V^{i}=0
$$

where $V$ is the tension field defined with respect to $g$ and $\gamma$ (cf. equation (2.7)),

2) $g-\gamma$ is $L^{2}$-perpendicular to the tangent space $T_{\gamma} \mathcal{N}$ of $\mathcal{N}$ at $\gamma$, in the sense of (4.5).

If this holds, we call $\gamma$ the shadow metric of $g$.

The following lemma, proved in appendix A, shows that the shadow metric condition can always be satisfied locally.

Lemma 4.3. Let $\mathcal{N}$ be the deformation space defined with respect to $\gamma_{0}$. Assume $\mathcal{N}$ is integrable. Let $s>n / 2+1$. There is a $\delta>0$ such that for $g \in \mathcal{M}$ satisfying $\left\|g-\gamma_{0}\right\|_{H^{s}}<\delta$, there exists a unique $\gamma$ in $\mathcal{N}$ satisfying $\|g-\gamma\|_{H^{s}}<2 \delta$, such that $\gamma$ is the shadow metric of $g$.

Let $\mathcal{P}$ be the shadow map introduced in remark 4.1. For $g \in \mathcal{M}$ close to $\gamma_{0}$, such that $\mathcal{P}$ is well defined near $g$, define the operator $Q_{g, \gamma}$ acting on tensors $z$ by

$$
\left(Q_{g, \gamma} z\right)^{i}=\left.\left.g^{m n} D \Gamma_{m n}^{i}\right|_{\gamma} \cdot D \mathcal{P}\right|_{g} . z
$$


The following lemma will be needed in the proof of local existence (see section 5), as well as in the proof of Lemma 6.1.

Lemma 4.4. Let $s>n / 2+1$. Let $\gamma_{0} \in \mathcal{E}_{\alpha}$ be given; assume $\gamma_{0}$ has stable, integrable deformation space $\mathcal{N}$; and for $g \in \mathcal{M}$ with shadow metric $\gamma \in \mathcal{N}$, let $Q_{g, \gamma}$ be defined by (4.7). There is a $\delta>0$ such that if $\left\|g-\gamma_{0}\right\|_{H^{s}}<\delta$, the inequality

$$
\left\|Q_{g, \gamma} z\right\|_{H^{s}} \leq C\left(\|g-\gamma\|_{H^{s}}+\left\|\gamma-\gamma_{0}\right\|_{H^{s}}\right)\|z\|_{H^{s-1}}
$$

holds.

Proof. We have

$$
\begin{aligned}
\left(Q_{g, \gamma} z\right)^{i}= & \left.\left.\left(g^{m n}-\gamma^{m n}\right) D \Gamma_{m n}^{i}\right|_{\gamma} \cdot D \mathcal{P}\right|_{g} \cdot z \\
& +\left.\left.\gamma^{m n} D \Gamma_{m n}^{i}\right|_{\gamma} \cdot D \mathcal{P}\right|_{g} \cdot z .
\end{aligned}
$$

By the discussion in section $2.3,\left.D \mathcal{P}\right|_{g} . z$ is of the form

$$
h^{\mathrm{TT} \|}+\mathcal{L}_{Y \|} \gamma
$$

A calculation shows that

$$
\left.\gamma^{m n} D \Gamma_{m n}^{i}\right|_{\gamma} \cdot u^{\mathrm{TT}}=0
$$

for any tensor $u^{\mathrm{TT}}$ which is $\mathrm{TT}$ with respect to $\gamma$. In particular, $\left.\gamma^{m n} D \Gamma_{m n}^{i}\right|_{\gamma} \cdot h^{\mathrm{TT} \|}=0$. Further, $Y^{\|}$solves (2.13). As discussed in section 2.2, the operator $P$ occurring in that equation is an isomorphism; in fact, as an operator $H^{s^{\prime}} \rightarrow H^{s^{\prime}-2}$ for any $s^{\prime}$.

This allows us to estimate

$$
\left\|\left.\gamma^{m n} D \Gamma_{m n}^{i}\right|_{\gamma} .\left.D \mathcal{P}\right|_{g} . z\right\| H_{H^{s}} \leq C\left\|\gamma-\gamma_{0}\right\|_{H^{s}}\|z\|_{H^{s-1}} .
$$

Here we have made use of the fact that the shadow map $\mathcal{P}$, in view of its definition, is smoothing; see remark 4.1. Together with the above discussion, this completes the proof.

q.e.d.

\subsection{The scale invariant evolution equations. Define}

$$
\delta_{i j}=\frac{1}{2}\left(\nabla_{i} V_{j}+\nabla_{j} V_{i}\right),
$$

where $V$ is the tension field defined with respect to $g$, $\gamma$. Following the work in [2], we will consider the modified Einstein evolution equations obtained by replacing $R_{i j}$ by the quasilinear elliptic system

$$
R_{i j}-\delta_{i j} .
$$

We remark that both $R_{i j}$ and $\delta_{i j}$ are scale invariant quantities. The modified Einstein evolution equations that we will consider are, in terms 
of the scale invariant variables,

$$
\begin{aligned}
g_{i j, T}= & 2 N \Sigma_{i j}+2\left(\frac{N}{n}-1\right) g_{i j}-\mathcal{L}_{X} g_{i j}, \\
\Sigma_{i j, T}= & -(n-1) \Sigma_{i j}-N\left(R_{i j}-\delta_{i j}+\frac{n-1}{n^{2}} g_{i j}\right) \\
& +\nabla_{i} \nabla_{j} N+2 N \Sigma_{i m} \Sigma_{j}^{m} \\
& -\frac{1}{n}\left(\frac{N}{n}-1\right) g_{i j}-(n-2)\left(\frac{N}{n}-1\right) \Sigma_{i j} \\
& -\mathcal{L}_{X} \Sigma_{i j}
\end{aligned}
$$

and the constraint equations take the form

$$
\begin{aligned}
& 0=R+\frac{n-1}{n}-|\Sigma|^{2}, \\
& 0=\nabla^{i} \Sigma_{i j} .
\end{aligned}
$$

The defining equations for the rescaled lapse and shift are

$$
\begin{aligned}
-\Delta N+\left(|\Sigma|^{2}+\frac{1}{n}\right) N= & 1, \\
\Delta X^{i}+R_{j}^{i} X^{j}-\mathcal{L}_{X} V^{i}= & \left(-2 N \Sigma^{m n}+2 \nabla^{m} X^{n}\right)\left(\Gamma[g]_{m n}^{i}-\Gamma[\gamma]_{m n}^{i}\right) \\
& -2\left(\frac{N}{n}-1\right) V^{i} \\
& +2\left(\nabla^{m} N\right) \Sigma_{m}^{i}+(2-n) \nabla^{i}\left(\frac{N}{n}-1\right) \\
& -g^{m n} \partial_{T} \Gamma[\gamma]_{m n}^{i},
\end{aligned}
$$

where the last term will be present only in case $\gamma_{0}$ has a nontrivial deformation space $\mathcal{N}$.

We remark that the evolution equations (4.9) do not automatically leave invariant the conditions $\operatorname{tr} \Sigma=0$ and $V=0$, but these hold as a consequence of imposing the defining equations (4.11); cf. the discussion in section 5 .

4.5. Evolution of the shadow metric. Recall from section 4.3 that the shadow metric is defined by the relation

$$
0=\int_{M}\left(g_{i j}-\gamma_{i j}\right) \frac{\partial \gamma^{i j}}{\partial q^{\alpha}} \mu_{\gamma}, \quad \alpha=1, \ldots, m .
$$

We now time differentiate (4.12). Note that we can write

$$
\partial_{T} \gamma_{i j}=\frac{\partial \gamma_{i j}}{\partial q^{\beta}} \dot{q}^{\beta}
$$


Thus time differentiating (4.12) gives

$$
\begin{aligned}
0= & \int_{M}\left(g_{i j}-\gamma_{i j}\right) \frac{\partial}{\partial q^{\beta}}\left(\frac{\partial \gamma^{i j}}{\partial q^{\alpha}}\right) \dot{q}^{\beta} \mu_{\gamma} \\
& +\int_{M}\left(g_{i j}-\gamma_{i j}\right) \frac{\partial \gamma^{i j}}{\partial q^{\alpha}} \frac{1}{2} \gamma^{m n} \frac{\partial \gamma_{m n}}{\partial q^{\beta}} \dot{q}^{\beta} \mu_{\gamma} \\
& +\int_{M} \partial_{T} g_{i j} \frac{\partial \gamma^{i j}}{\partial q^{\alpha}} \mu_{\gamma} \\
& +\int_{M} \dot{q}^{\beta}\left(\frac{\partial \gamma_{i j}}{\partial q^{\beta}} \gamma^{i m} \gamma^{j n} \frac{\partial \gamma_{m n}}{\partial q^{\alpha}}\right) \mu_{\gamma} .
\end{aligned}
$$

The matrix

$$
M_{\alpha \beta}:=\int_{M} \frac{\partial \gamma_{i j}}{\partial q^{\beta}} \gamma^{i m} \gamma^{j n} \frac{\gamma_{m n}}{\partial q^{\alpha}} \mu_{\gamma}
$$

is invertible since the $\frac{\partial \gamma_{i j}}{\partial q^{\alpha}}$ form a basis for the tangent space $T_{\gamma} \mathcal{N}$.

Thus for $\left(g_{i j}-\gamma_{i j}\right)$ sufficiently small,

$$
\begin{aligned}
\tilde{M}_{\alpha \beta}:= & \int_{M}\left(\frac{\partial \gamma_{i j}}{\partial q^{\beta}} \gamma^{i m} \gamma^{j n} \frac{\partial \gamma_{m n}}{\partial q^{\alpha}}\right) \mu_{\gamma} \\
& +\int_{M}\left(g_{i j}-\gamma_{i j}\right) \frac{\partial}{\partial q^{\beta}}\left(\frac{\partial \gamma^{i j}}{\partial q^{\alpha}}\right) \mu_{\gamma} \\
& +\int_{M}\left(g_{i j}-\gamma_{i j}\right) \frac{\partial \gamma^{i j}}{\partial q^{\alpha}} \frac{1}{2} \gamma^{m n} \frac{\partial \gamma_{m n}}{\partial q^{\beta}} \mu_{\gamma}
\end{aligned}
$$

will also be invertible and positive definite so we can solve for $\dot{q}^{\beta}$ in terms of the inverse matrix applied to $\int_{M} \partial_{T} g_{i j} \frac{\partial \gamma^{i j}}{\partial q^{\alpha}} d \mu_{\gamma}$. The defining equation for $\partial_{T} \gamma_{i j}$ can now be expressed in terms of an equation for $\dot{q}^{\beta}$ of the form

$$
0=\tilde{M}_{\alpha \beta} \dot{q}^{\beta}+\int_{M} \partial_{T} g_{i j} \frac{\partial \gamma^{i j}}{\partial q^{\alpha}} \mu_{\gamma} .
$$

In applying this setup, $\partial_{T} g_{i j}$ will be given by (4.9a).

Remark 4.5. In terms of the shadow map $\mathcal{P}: \mathcal{M} \rightarrow \mathcal{N}$ (cf. remark 4.1), we have

$$
\partial_{T} \gamma=\left.D \mathcal{P}\right|_{g}, \partial_{T} g
$$

\section{Local Existence}

We shall prove local wellposedness for the system $(4.9,4.10,4.11)$ for the rescaled variables $(g, \Sigma, N, X)$ by making use of the results of [2] applied to the corresponding system of modified Einstein evolution equations, constraint equations, and defining equations for the original variables $(\tilde{g}, \tilde{K}, \tilde{N}, \tilde{X})$. Note that in $[\mathbf{2}]$ these fields are denoted without the tilde. 
If the deformation space is trivial, i.e. if $\gamma_{0}$ is strictly stable, we can apply the same argument as in the standard case considered in [2]. We will now consider the modifications necessary for the case when the deformation space is nontrivial. Let $t$ be the time variable in the system considered in $[\mathbf{2}]$. For the solution to this system, it will be the case that $t$ is the CMC time, $t=\operatorname{tr} K$. However, it should be noted that in the course of the iteration procedure, and the proof of wellposedness, as presented in $[\mathbf{2}]$, this cannot be assumed. We remark that it follows from our assumption on initial data, and continuity, that $\tilde{g}^{i j} \tilde{K}_{i j} / t$ is close to 1 for times $t$ close to the initial time, so that in the construction of solutions we may assume that we are in an almost CMC situation.

For the application in this paper, we are interested only in the small data situation where $t^{2} \tilde{g}$ is close to a background metric $\gamma_{0}$, and $t \tilde{K}_{i j}-$ $\left(t^{2} / n\right) \tilde{g}_{i j}$ is small.

Define $\bar{\tau}$ to be the average mean curvature,

$$
\bar{\tau}=\left(\int_{M} \tilde{g}^{i j} \tilde{K}_{i j} \mu_{\tilde{g}}\right) / \int_{M} \mu_{\tilde{g}} .
$$

The system of equations which will be considered is the same as in [2], with the difference that the shadow metric $\gamma$ is time dependent, $\gamma=\gamma(t)$, and that the spatially harmonic gauge is now defined with respect to the time dependent shadow metric $\gamma$. We define the shadow metric using the shadow map $\mathcal{P}$ (see remark 4.1), by letting the shadow metric $\gamma(t)$ be given by

$$
\gamma(t)=\mathcal{P}\left[\bar{\tau}^{2}(t) \tilde{g}(t)\right]
$$

As mentioned above, we are considering a small data situation, and in particular, $\bar{\tau}^{2} \tilde{g}$ is in a neighborhood of the shadow manifold $\mathcal{N}$ where the map $\mathcal{P}$ is well defined and smooth.

Using the notation of [2], the system of evolution equations can be written in the form

$$
L[\tilde{g}, \tilde{N}, \tilde{X}] \mathcal{U}=\mathcal{F}
$$

with $\mathcal{U}=(u, v)$. Let $\nabla[\gamma]$ be the covariant derivative defined with respect to the metric $\gamma$. In order to prove local wellposedness for the resulting system, we use a wave-equation type energy analogous to the one used in [2], i.e. an energy of the form

$$
E=\int_{M}\left(|u|^{2}+|\nabla[\gamma] u|_{\tilde{g}}^{2}+|v|^{2}\right) \mu_{\tilde{g}}
$$

where for a 2-tensor $u,|u|$ is defined in terms of $\gamma$ by $|u|^{2}=u_{i j} u_{k l} \gamma^{i k} \gamma^{j l}$, and $|\nabla[\gamma] u|_{\tilde{g}}$ is defined by $|\nabla[\gamma] u|_{\tilde{g}}^{2}=\nabla[\gamma]_{i} u_{j k} \nabla[\gamma]_{l} u_{m n} \tilde{g}^{i l} \gamma^{j m} \gamma^{k n}$.

Given $\tilde{g}, \tilde{X}$, let $\rho$ be defined by

$$
\rho=-\frac{1}{2}\left(\partial_{t} \tilde{g}-\mathcal{L}_{\tilde{X}} \tilde{g}\right) .
$$


Taking into account the time dependence of $\gamma$, the energy estimate $[\mathbf{2}$, lemma 2.4] is replaced by

$$
\partial_{t} E \leq C\left(E^{1 / 2}\|\mathcal{F}\|_{H^{1} \times L^{2}}+\left(1+\|\rho\|_{L^{\infty}}+\left\|\partial_{t} \gamma\right\|_{L^{\infty}}\right) E\right)
$$

Using this estimate, it is straightforward to prove the higher order energy estimates needed for local wellposedness, following the same argument as in [2]. Once the energy estimate is obtained, a solution to the field equations is constructed using an iteration as in $[\mathbf{2}, \S 2.2]$.

The argument from $[\mathbf{2}]$ carries over nearly without modification. However, the fact that we now allow for a moving shadow metric $\gamma$ gives rise to an extra term in the defining equation for the shift vector, of the form

$$
\tilde{g}^{m n} \partial_{t} \Gamma[\gamma]_{m n}^{i}
$$

Since $\partial_{t} \gamma=\left.D \mathcal{P}\right|_{\bar{\tau}^{2} \tilde{g}} \partial_{t}\left(\bar{\tau}^{2} \tilde{g}\right)$, and $\partial_{t} \tilde{g}=-2 \tilde{N} \tilde{K}+\mathcal{L}_{\tilde{X}} \tilde{g}$, this adds a nonlocal operator acting on $\tilde{X}$ to the defining equation for the shift vector.

However, in view of the estimate given in Lemma 4.4, the effect of this term is a small perturbation for the small data situation we are considering here, and hence the modified shift equation satisfies the same estimates as the one which was considered in [2]. In order to analyze the modified shift operator, it suffices to consider the expression

$$
\left.\left.\tilde{g}^{m n} D \Gamma_{m n}^{i}\right|_{\gamma} \cdot D \mathcal{P}\right|_{\bar{\tau}^{2} \tilde{g}}\left(\mathcal{L}_{\bar{\tau} \tilde{X}} \bar{\tau}^{2} \tilde{g}\right)
$$

Here and below we have made use of the average mean curvature to introduce in appropriate places scale invariant fields along the lines of section 4.1. We shall apply Lemma 4.4 to estimate

$$
Q_{\left(\bar{\tau}^{2} \tilde{g}, \gamma\right)}^{i} \cdot\left(\mathcal{L}_{\bar{\tau} \tilde{X}} \bar{\tau}^{2} \tilde{g}\right)=\left.\left.\bar{\tau}^{-2} \tilde{g}^{m n} D \Gamma_{m n}^{i}\right|_{\gamma} \cdot D \mathcal{P}\right|_{\bar{\tau}^{2} \tilde{g}}\left(\mathcal{L}_{\bar{\tau} \tilde{X}} \bar{\tau}^{2} \tilde{g}\right)
$$

We have, in the small data situation we are considering,

$$
\begin{aligned}
\left\|Q_{\left(\bar{\tau}^{2} \tilde{g}, \gamma\right)} \cdot \mathcal{L}_{\bar{\tau} \tilde{X}} \bar{\tau}^{2} \tilde{g}\right\|_{H^{s}} & \leq C\left(\left\|\bar{\tau}^{2} \tilde{g}-\gamma\right\|_{H^{s}}+\left\|\gamma-\gamma_{0}\right\|_{H^{s}}\right)\left\|\mathcal{L}_{\bar{\tau} \tilde{X}} \bar{\tau}^{2} \tilde{g}\right\|_{H^{s-1}} \\
(5.2) & \leq C\left(\left\|\bar{\tau}^{2} \tilde{g}-\gamma\right\|_{H^{s}}+\left\|\gamma-\gamma_{0}\right\|_{H^{s}}\right)\|\bar{\tau} \tilde{X}\|_{H^{s}} .
\end{aligned}
$$

Note we are considering only the case where $t<t_{0}<0$ for some $t_{0}$. Consequently, due to the small data assumption, we may assume without loss of generality that $2 \bar{\tau}_{*}<\bar{\tau}<\bar{\tau}_{*}<0$ for some $\bar{\tau}_{*}$. In view of the estimate (5.2), the extra term in the shift equation due to the time dependence of $\gamma$ can be considered as a small perturbation which does not affect the existence and uniqueness results for this equation proved in [2]. In particular, the estimates for $\tilde{X}$ needed for the iteration argument in $[\mathbf{2}]$ are valid also for the modified system.

With this remark, the rest of the argument goes through unchanged. This proves local wellposedness in $H^{s}, s>n / 2+1$. In particular (see [2, theorem 3.1]), for initial data $\left(\tilde{g}_{0}, \tilde{K}_{0}\right) \in H^{s} \times H^{s-1}$ at $t_{0}$ satisfying the small data conditions as above, we have a solution curve

$$
t \mapsto(\tilde{g}, \tilde{K}, \tilde{X}, \tilde{N}) \in H^{s} \times H^{s-1} \times H^{s+1} \times H^{s+1},
$$


defined on an open time interval containing $t_{0}$.

It remains to consider the propagation of gauges and constraints. Introduce, as in $[\mathbf{2}]$, the gauge and constraint quantities

$$
\begin{aligned}
\tilde{A} & =\operatorname{tr}_{\tilde{g}} \tilde{K}-t, \\
\tilde{V}^{k} & =\tilde{g}^{i j}\left(\Gamma[\tilde{g}]_{i j}^{k}-\Gamma[\gamma]_{i j}^{k}\right), \\
\tilde{F} & =R[\tilde{g}]+\left(\operatorname{tr}_{\tilde{g}} \tilde{K}\right)^{2}-|\tilde{K}|_{\tilde{g}}^{2}-\nabla[\tilde{g}]_{i} \tilde{V}^{i}, \\
\tilde{D}_{i} & =\nabla[\tilde{g}]_{i} \operatorname{tr}_{\tilde{g}} \tilde{K}-2 \nabla[\tilde{g}]^{j} \tilde{K}_{j i} .
\end{aligned}
$$

Here we raise and lower indices using $\tilde{g}^{i j}$ and $\tilde{g}_{i j}$ and use the notation $\operatorname{tr}_{\tilde{g}} \tilde{K}=\tilde{g}^{m n} \tilde{K}_{m n}$ and $|\tilde{K}|_{\tilde{g}}^{2}=\tilde{K}_{m n} \tilde{K}^{m n}$. We consider the energy expression $\widetilde{\mathcal{E}}$ defined by

$\widetilde{\mathcal{E}}=\frac{1}{2} \int_{M}\left(|\tilde{A}|^{2}+|\nabla[\tilde{g}] \tilde{A}|_{\tilde{g}}^{2}+|\tilde{F}|^{2}\right) \mu_{\tilde{g}}+\frac{1}{2} \int_{M}\left(|\tilde{V}|_{\tilde{g}}^{2}+|\nabla[\tilde{g}] \tilde{V}|_{\tilde{g}}^{2}+|\tilde{D}|_{\tilde{g}}^{2}\right) \mu_{\tilde{g}}$.

Recall that the defining equations for $\tilde{N}$ and $\tilde{X}$ are derived by time differentiating the gauge conditions using the evolution equations. In particular, the shift equation may be chosen such that the expression for $\partial_{t} \tilde{V}$ is the same as in the rigid case. One sees from considering the gauge and constraint quantities that the only potential difference from the case when the deformation space is trivial is via the evolution equation for $\tilde{V}$. Thus, with the aforementioned choice, the evolution equations for the gauge and constraint quantities are identical, in the case when the deformation space is nontrivial, to the evolution equations valid in the case when the background metric $\gamma_{0}$ is rigid. Therefore, we are able to conclude by the same argument as in $[\mathbf{2}]$ that if the constraint and gauge conditions are satisfied initially, they are also satisfied throughout the course of the evolution.

The system discussed here is the modified Einstein evolution equations without rescaling. We now return to the situation considered in the rest of the paper and state the results we have proved for the rescaled variables $(g, \Sigma, N, X)$ introduced in section 4 . It follows from the above discussion that the system of equations for the rescaled variables (4.9, $4.10,4.11)$ is also well posed in the shadow metric gauge. As shown in $[2]$, the result that we have proved can be formulated as a continuation principle, which will be used for the global existence theorem.

In formulating the continuation principle, we will deal with rescaled data. Fix a background metric $\gamma_{0} \in \mathcal{N}$, and for $\delta>0, s>n / 2+1$, let $\mathcal{B}_{s, \delta}\left(\gamma_{0}, 0\right)$ be the ball of radius $\delta$ in $\mathcal{C}^{s}$, centered on $\left(\gamma_{0}, 0\right)$. Here $\mathcal{C}^{s}$ denotes the space of $(g, \Sigma) \in \mathcal{C}$ such that $(g, \Sigma) \in H^{s} \times H^{s-1}$. The following lemma follows immediately from Lemma 4.3 and the construction of the shadow map $\mathcal{P}$.

Lemma 5.1. Assume $\mathcal{N}$ is integrable near $\gamma_{0}$. Then there is a $\delta>0$ such that 
1) for $(g, \Sigma) \in \mathcal{B}_{s, \delta}\left(\gamma_{0}, 0\right)$, there is a unique $\gamma \in \mathcal{N}$ such that the triple $(\gamma, g, \Sigma)$ satisfies the shadow metric condition,

2) there is a constant $C$ such that the Frechet derivative DP satisfies

$$
\|D \mathcal{P}\|_{O p, s, \infty} \leq C
$$

in $\mathcal{B}_{s, \delta}\left(\gamma_{0}, 0\right)$. Here the left hand side is the operator norm of the Frechet derivative from $H^{s}$ to $L^{\infty}$.

Based on the above discussion of the proof of local wellposedness and the continuation principle [2, theorem 5.1], we can state the following continuation principle which is appropriate for the situation considered in this paper. Note that in the small data situation considered here, the assumption of negative sectional curvature for the target metric made in $[2]$ is not needed. In particular, if $\gamma$ is negative Einstein and $g$ is close to $\gamma$, then the operator $X^{i} \mapsto g^{m n} R[\gamma]^{i}{ }_{m j n} X^{j}$ is strictly negative, in which case the proof of $[\mathbf{2}$, lemma 5.2] goes through unchanged.

Theorem 5.2. Fix $s>n / 2+1$. Let $\gamma_{0} \in \mathcal{N}$ be given and assume $\mathcal{N}$ is integrable near $\gamma_{0}$. Then there is a $\delta_{0}>0$ such that the conclusion of Lemma 5.1 holds, and such that the following continuation principle holds for the system $(4.9,4.10,4.11)$ :

Let $\left(g_{0}, \Sigma_{0}\right)$ be rescaled data given at an initial rescaled time $T_{0}$, such that the triple $\left(\gamma_{0}, g_{0}, \Sigma_{0}\right)$ satisfies the shadow metric condition. Assume that

1) $\left(g_{0}, \Sigma_{0}\right) \in \mathcal{B}_{s, \delta_{0}}\left(\gamma_{0}, 0\right)$,

2) $\left[T_{0}, T_{+}\right)$is the maximal existence interval in $H^{s}$ for the system $(4.9,4.10,4.11)$ with initial data $\left(\gamma_{0}, g_{0}, \Sigma_{0}\right)$.

Then, either

1) $T_{+}=\infty$ or

2) the solution curve $T \mapsto\left(g_{i j}(T), \Sigma_{i j}(T)\right)$ leaves $\mathcal{B}_{s, \delta_{0}}\left(\gamma_{0}, 0\right)$ at some finite time.

\section{Definition of Energies}

In this section, assume that a background $\gamma_{0}$ is given, with integrable deformation space $\mathcal{N}$, and let $\delta_{0}>0$ be a sufficiently small constant such that for $(g, \Sigma) \in B_{s, \delta_{0}}\left(\gamma_{0}, 0\right)$, the conclusion of Theorem 5.2 holds. For the estimates proved below in this section, we suppose that $\delta_{0}$ is decreased as necessary.

6.1. Small quantities. The data corresponding to the Lorentz cone metric (4.1) is

$$
(g, \Sigma, N, X)=(\gamma, 0, n, 0)
$$

Thus,

$$
g-\gamma, \Sigma, \frac{N}{n}-1, X
$$


should be considered as small quantities in the small data situation we are considering.

In this section, we shall consider a solution to the rescaled Einstein equations presented above, satisfying the CMCSH gauge conditions (4.6) with respect to a curve of background metrics $\gamma$ defined by the shadow metric condition. In case $\gamma_{0}$ is strictly stable, $\gamma \equiv \gamma_{0}$ and hence $\partial_{T} \gamma=0$. We will show that in this case the quantities $N / n-1, X$ satisfy quadratic estimates in terms of $g-\gamma, \Sigma$.

If $\gamma_{0}$ has a nontrivial integrable deformation space, then

$$
\partial_{T} \gamma=h^{\mathrm{TT} \|}+\mathcal{L}_{Y \|} \gamma
$$

is in general non-vanishing, and in particular $Y^{\|} \neq 0$. In this case, $N / n-1$ satisfies a quadratic estimate as in the strictly stable case, while $X, Y^{\|}$separately cannot be expected to satisfy such an estimate. However, as we shall prove, the sum $X+Y^{\|}$does. This is precisely what is needed for the energy estimates to go through.

Lemma 6.1. Let $s>n / 2+1$. For $(g, \Sigma) \in B_{s, \delta_{0}}\left(\gamma_{0}, 0\right)$, there is a constant $C>0$ such that the inequalities

$$
\begin{gathered}
\left\|\frac{N}{n}-1\right\|_{H^{s+1}} \leq C\|\Sigma\|_{H^{s-1}}^{2}, \\
\|X\|_{H^{s+1}} \leq C\left(\|\Sigma\|_{H^{s-1}}+\|g-\gamma\|_{H^{s}}\right)
\end{gathered}
$$

and

$$
\left\|X+Y^{\|}\right\|_{H^{s+1}} \leq C\left(\|\Sigma\|_{H^{s-1}}^{2}+\|g-\gamma\|_{H^{s}}^{2}\right)
$$

hold.

Proof. Recall that the scale invariant lapse and shift $N, X^{i}$ solve the defining equations (4.11). It follows from the lapse equation (4.11a) that $\hat{N}=N / n-1$ satisfies the equation

$$
-\Delta \hat{N}+\left(|\Sigma|^{2}+\frac{1}{n}\right) \hat{N}=-|\Sigma|^{2} .
$$

From this, one finds that if the gauge conditions are satisfied, $\hat{N}$ can be estimated in terms of the square norm of $\Sigma$, which proves (6.2).

In case $\gamma_{0}$ is rigid, then $\partial_{T} \gamma=0$ and $X$ can be estimated in terms of square norms of $g-\gamma$ and $\Sigma$; cf. [2, $\S 3]$. However, in case $\gamma_{0}$ has a nontrivial deformation space $\mathcal{V}, \partial_{T} \gamma$ will in general be nonzero. By (4.9a) and (6.2), we have an estimate of the form

$$
\left\|\partial_{T} g\right\|_{H^{s-1}} \leq C\left(\|\Sigma\|_{H^{s-1}}+\|X\|_{H^{s}}\right) .
$$

As discussed in section 2, we may without loss of generality assume that $\gamma_{0}$, and all metrics in $\mathcal{N}$ are smooth. Hence, it follows from the 
definition of the shadow metric that $\partial_{T} \gamma$ is smooth and that an estimate of the form

$$
\left\|\partial_{T} \gamma\right\|_{H^{s^{\prime}}} \leq C\left\|\partial_{T} g\right\|_{L^{2}}
$$

holds for any $s^{\prime} \geq 0$. Consider the last term in equation (4.11b). Following the proof of Lemma 4.4, it is straightforward in view of the above discussion to show that an inequality of the form

$$
\begin{aligned}
&\left\|g^{m n} \partial_{T} \Gamma[\gamma]_{m n}^{i}\right\|_{H^{s}} \leq \\
& C\left(\|g-\gamma\|_{H^{s}}+\left\|\gamma-\gamma_{0}\right\|_{H^{s}}\right)\left(\|\Sigma\|_{H^{s-1}}+\|X\|_{H^{s}}\right)
\end{aligned}
$$

holds.

We now recall some facts from $[\mathbf{2}]$. Let $V_{g, \gamma}$ be the tension field defined with respect to the metrics $g, \gamma$, and denote by $\nabla[g], \Delta[g], R[g]$ the covariant derivative, Laplacian, and curvature defined with respect to $g$, and by $\nabla[\gamma], \Delta[\gamma], R[\gamma]$ the corresponding objects defined with respect to $\gamma$.

Let $\Delta_{g, \gamma}$ be the operator defined on symmetric 2 -tensors by

$$
\Delta_{g, \gamma} h_{i j}=\frac{1}{\mu_{g}} \nabla[\gamma]_{m}\left(g^{m n} \mu_{g} \nabla[\gamma]_{n} h_{i j}\right)
$$

(see $[\mathbf{2},(1.8)]$ ). Define the operator $P_{g, \gamma}$ by

$P_{g, \gamma} X^{i}=\Delta[g] X^{i}+R[g]_{j}^{i} X^{j}-\mathcal{L}_{X} V_{g, \gamma}^{i}-2\left(\nabla[g]^{m} X^{n}\right)\left(\Gamma[g]_{m n}^{i}-\Gamma[\gamma]_{m n}^{i}\right)$.

The defining equation for $X$ is given in terms of $P_{g, \gamma}$ in equation (6.10) below. Note that we are interested here in the case where $V=0$, but we include it in the above formula since it makes the calculations below more transparent. Similarly, let $P_{\gamma, \gamma}$ be the corresponding operator with $g$ replaced by $\gamma$

$P_{\gamma, \gamma} X^{i}=\Delta[\gamma] X^{i}+R[\gamma]_{j}^{i} X^{j}-\mathcal{L}_{X} V_{\gamma, \gamma}^{i}-2\left(\nabla[\gamma]^{m} X^{n}\right)\left(\Gamma[\gamma]_{m n}^{i}-\Gamma[\gamma]_{m n}^{i}\right)$

where the last two terms vanish identically. By the results of $[\mathbf{2}, \S 5]$, these operators take the form

$$
\begin{aligned}
& P_{g, \gamma} X^{i}=g^{m n} \nabla[\gamma]_{m} \nabla[\gamma]_{n} X^{i}+g^{m n} R[\gamma]_{m j n}^{i} X^{j} \\
& P_{\gamma, \gamma} X^{i}=\gamma^{m n} \nabla[\gamma]_{m} \nabla[\gamma]_{n} X^{i}+\gamma^{m n} R[\gamma]_{m j n}^{i} X^{j}
\end{aligned}
$$

where the index on $R[\gamma]$ is raised with $\gamma$. By the discussion in section 2.2, $P_{g, \gamma}: H^{s+1} \rightarrow H^{s-1}$ is an isomorphism, as is $P_{\gamma, \gamma}$. From this it is straightforward to show that the inequality

$$
\|X\|_{H^{s+1}} \leq C\left(\|\Sigma\|_{H^{s-1}}+\|g-\gamma\|_{H^{s}}\right)
$$

holds, i.e. we have proved (6.3). Here we have made use of (6.6) and absorbed terms which can be estimated by

$$
\left(\|g-\gamma\|_{H^{s}}+\left\|\gamma-\gamma_{0}\right\|_{H^{s}}\right)\|X\|_{H^{s+1}}
$$

after a small change in the constant. 
It remains to prove (6.4). We write the defining equation for $X$ as

$$
\begin{aligned}
P_{g, \gamma} X^{i}= & -2 N \Sigma^{m n}\left(\Gamma[g]_{m n}^{i}-\Gamma[\gamma]_{m n}^{i}\right)-2\left(\frac{N}{n}-1\right) V^{i} \\
& +2\left(\nabla^{m} N\right) \Sigma_{m}^{i}+(2-n) \nabla^{i}\left(\frac{N}{n}-1\right) \\
& -g^{m n} \partial_{T} \Gamma[\gamma]_{m n}^{i}
\end{aligned}
$$

where by the gauge conditions we may set $V=0$. All terms in the right hand side of (6.10) are quadratic in small quantities, except the last.

Recall that $\partial_{T} \gamma$ is of the form (6.1). A calculation shows

$$
\gamma^{m n} D \Gamma[\gamma]_{m n}^{i} \cdot h^{\mathrm{TT} \|}=0
$$

due to the fact that $h^{\mathrm{TT} \|}$ is transverse traceless with respect to $\gamma$. Therefore, we have

$$
\gamma^{m n} \partial_{T} \Gamma[\gamma]_{m n}^{i}=\gamma^{m n} D \Gamma[\gamma]_{m n}^{i} \cdot\left(\mathcal{L}_{Y \|} \gamma\right)
$$

and a direct calculation gives

$$
\gamma^{m n} \partial_{T} \Gamma[\gamma]_{m n}^{i}=\Delta[\gamma] Y^{\| i}+R[\gamma]_{f}^{i} Y^{\| f}
$$

Comparing with (6.9) we have

$$
\gamma^{m n} \partial_{T} \Gamma[\gamma]_{m n}^{i}=P_{\gamma, \gamma} Y^{\| i}
$$

and hence we may write the defining equation for $X$ in the form

$$
\begin{aligned}
P_{\gamma, \gamma}\left(X^{i}+Y^{\| i}\right)= & -2 N \Sigma^{m n}\left(\Gamma[g]_{m n}^{i}-\Gamma[\gamma]_{m n}^{i}\right) \\
& +2\left(\nabla^{m} N\right) \Sigma_{m}^{i}+(2-n) \nabla^{i}\left(\frac{N}{n}-1\right) \\
& -\left(g^{m n}-\gamma^{m n}\right)\left(\nabla[\gamma]_{m} \nabla[\gamma]_{n} X^{i}+R[\gamma]^{i}{ }_{m j n} X^{j}\right) \\
& -\left(g^{m n}-\gamma^{m n}\right) \partial_{T} \Gamma[\gamma]_{m n}^{i}
\end{aligned}
$$

where we have set $V=0$. Estimating each term gives, after making use of (6.2) and (6.3), as well as of the elementary inequality $a b \leq \frac{1}{2}\left(a^{2}+b^{2}\right)$, the estimate (6.4).

q.e.d.

6.2. Splitting the Einstein equations. In this section we will, in preparation for proving the energy estimates needed for the proof of our main result, rewrite the scale invariant Einstein along the lines of [2]. We shall need the following estimate for the curvature term in (4.9b). Let $\mathcal{L}_{g, \gamma}$ be the operator on symmetric 2 -tensors defined by

$$
\mathcal{L}_{g, \gamma} h=-\Delta_{g, \gamma} h-2 \stackrel{\circ}{R}_{\gamma} h .
$$

Here $\stackrel{\circ}{R}_{\gamma} h$ is given by (2.2) with the curvature tensor $R[\gamma]$. In particular, if $g=\gamma$, then $\mathcal{L}_{g, \gamma}$ coincides with the operator $\mathcal{L}$ defined by (2.1). The following lemma follows from the form of $R_{i j}$ derived in the proof of $[\mathbf{2}$, theorem 3.1]. 


\section{Lemma 6.2.}

$$
R_{i j}-\delta_{i j}+\frac{(n-1)}{n^{2}} g_{i j}=\frac{1}{2} \mathcal{L}_{g, \gamma}(g-\gamma)_{i j}+J_{i j}
$$

where

$$
\|J\|_{H^{s-1}} \leq C\|g-\gamma\|_{H^{s}}^{2} .
$$

We will write the Einstein evolution equations in terms of variables $(u, v)$ defined by

$$
u=g-\gamma, \quad v=2 n \Sigma
$$

and the normalized lapse

$$
\omega=\frac{N}{n} .
$$

We use the identity

$$
\mathcal{L}_{X} u_{i j}=X^{m} \nabla[\gamma]_{m} u_{i j}+u_{i m} \nabla[\gamma]_{j} X^{m}+u_{m j} \nabla[\gamma]_{i} X^{m}
$$

to expand the Lie derivative. With these definitions, we have

Lemma 6.3. The Einstein evolution equations (4.9) are equivalent to the system

$$
\begin{aligned}
& \partial_{T} u=\omega v-h^{\mathrm{TT} \|}-X^{i} \nabla[\gamma]_{i} u+\mathcal{F}_{u}, \\
& \partial_{T} v=-(n-1) v-n^{2} \omega \mathcal{L}_{g, \gamma} u-X^{i} \nabla[\gamma]_{i} v+\mathcal{F}_{v}
\end{aligned}
$$

where

$$
\begin{aligned}
\left\|\mathcal{F}_{u}\right\|_{H^{s}} & \leq C\left(\|u\|_{H^{s}}^{2}+\|v\|_{H^{s-1}}^{2}\right), \\
\left\|\mathcal{F}_{v}\right\|_{H^{s-1}} & \leq C\left(\|u\|_{H^{s}}^{2}+\|v\|_{H^{s-1}}^{2}\right) .
\end{aligned}
$$

Proof. A direct calculation gives

$$
\begin{aligned}
\partial_{T} u= & \omega v-h^{\mathrm{TT} \|}+2(\omega-1) g-\mathcal{L}_{X} g-\mathcal{L}_{Y \|} \gamma \\
= & \omega v-h^{\mathrm{TT} \|}-\mathcal{L}_{X} u \\
& +2(\omega-1) g-\mathcal{L}_{X+Y} \| \gamma .
\end{aligned}
$$

We now expand the term $\mathcal{L}_{X} u$ using (6.13) and put $\partial_{T} u$ in the form (6.14a). The term $\mathcal{F}_{u}$ defined in this manner can be shown, using the inequalities of Lemma 6.1, to satisfy the estimate (6.15a).

For $v$, we proceed in a similar manner, making use of Lemma 6.2 to rewrite the curvature term in equation (4.9b).

q.e.d. 
6.3. The linearized Einstein equations. It is straightforward to linearize the system defined by equations (4.6), (4.10)-(4.12), and (6.14) about the exact solution $(g, \Sigma, N, X)=(\gamma, 0, n, 0)$ where $\gamma$ lies in the deformation space $\mathcal{N}$ of a fixed Einstein metric $\gamma_{0}$. Linearization of the Hamiltonian constraint (4.10a) and the gauge condition (4.6), together with the Einstein condition satisfied by $\gamma$, immediately imply that the first variation $\delta u$ of $u=g-\gamma$ is transverse traceless with respect to the background metric $\gamma$. Linearization of the momentum constraint (4.10b) together with the condition $g^{i j} \Sigma_{i j}=0$ implies that $\delta v_{i j}=2 n \delta \Sigma_{i j}$ is also transverse traceless with respect to $\gamma$. Variation of equations (4.11) leads to $\delta N=0$ and $\delta X^{i}+\delta Y^{\| i}=0$. From equation (2.13) one finds that $\delta Y^{\|}$ is determined from $\delta h^{\mathrm{TT}} \|$, which latter is also transverse traceless with respect to $\gamma$ and satisfies $\mathbb{P}^{\|} \delta h^{\mathrm{TT}}\left\|=\delta h^{\mathrm{TT}}\right\|$. Variation of the shadow metric condition (4.12) shows further that $\mathbb{P}^{\|}(\delta u)=0$, i.e., that the transverse traceless tensor $\delta u$ satisfies $\delta u=\delta u^{\perp}$.

It is now straightforward to linearize the evolution equations (6.14) and decompose them into $\|$ and $\perp$ projections. This leads immediately to $\delta v^{\|}=\delta h^{\mathrm{TT}} \|$ and to

$$
\begin{aligned}
\partial_{T} \delta u^{\perp} & =\delta v^{\perp} \\
\partial_{T} \delta v^{\|} & =-(n-1) \delta v^{\|} \\
\partial_{T} \delta v^{\perp} & =-(n-1) \delta v^{\perp}-n^{2} \mathcal{L} \delta u^{\perp}
\end{aligned}
$$

where $\mathcal{L}$ is the operator given by (6.11) with $g=\gamma$. These combine to give the second order equation

$$
\delta u_{, T T}^{\perp}+(n-1) \delta u_{, T}^{\perp}+n^{2} \mathcal{L} \delta u^{\perp}=0
$$

for $\delta u^{\perp}$ and to give immediately that

$$
\delta v^{\|}=\left.\delta v^{\|}\right|_{T=T_{0}} e^{-(n-1)\left(T-T_{0}\right)} .
$$

It follows that $\delta h^{\mathrm{TT}} \|=\delta v^{\|}$, and therefore also $\delta Y^{\|}$, all decay at the same universal exponential rate (at least in this linearized approximation). As we shall demonstrate below, cf. section 8, exponential decay also holds for the solution to the full nonlinear problem.

While equation (6.16) may be solved explicitly by separation of variables (as shown in [10] and recalled below), we shall need to prove energy estimates for this system in order to have a tool adequate for generalization to the nonlinear problem. In any case, recalling that we can write

$$
\delta \gamma=\delta q^{(\alpha)}\left(h^{(\alpha) \mathrm{TT} \|}+\mathcal{L}_{Y^{(\alpha)} \|} \gamma\right)
$$


where $h^{(\alpha) \text { TT } \|}$ and $Y^{(\alpha) \|}$ are background quantities and $\delta q^{(\alpha)}=\delta q^{(\alpha)}(T)$ are functions of $T$ only, we can decompose $\delta u=\delta g-\delta \gamma$ into its constituents and show that

$$
\delta q^{(\alpha)}(T)=-\left(\left.\partial_{T}\left(\delta q^{(\alpha)}\right)\right|_{T=T_{0}}\right) \frac{1}{n-1} e^{-(n-1)\left(T-T_{0}\right)}+\delta q^{(\alpha)}(\infty)
$$

where $\delta q^{(\alpha)}(\infty)$ is a constant of integration that yields the asymptotic value of $\delta \gamma$. The perturbed metric $\delta g$ is thus given by $\delta g=\delta u^{\perp}+\delta \gamma$, with $\delta u^{\perp}$ and $\delta \gamma$ determined as above.

In order to understand how to prove energy estimates for the system (6.16), we perform a separation of variables. Let $\lambda$ be a nonzero eigenvalue $\mathcal{L}$ and let $X$ be an eigentensor corresponding to $\lambda$. Equation (6.16) gives the model system

$$
\ddot{X}+(n-1) \dot{X}+n^{2} \lambda X=0,
$$

which we recognize as a damped oscillator equation. This has characteristic roots

$$
\frac{-(n-1) \pm \sqrt{(n-1)^{2}-4 n^{2} \lambda}}{2} .
$$

If $\lambda>\frac{(n-1)^{2}}{4 n^{2}}$, then the characteristic equation has a complex pair of roots with real part $-(n-1) / 2$, and hence there is a universal exponential rate of decay $-(n-1) / 2$. If $0<\lambda<\frac{(n-1)^{2}}{4 n^{2}}$ the characteristic equation has a pair of negative real roots. In this case we have an "anomalous" rate of decay depending on $\lambda$. If $\lambda=\frac{(n-1)^{2}}{4 n^{2}}$ we have a critically damped oscillator. We avoid dealing directly with this case, by decreasing $\lambda$ slightly.

6.4. Energies for the damped oscillator. The equation (6.17) is an ODE with constant coefficients and can therefore be analyzed by elementary means. However, since the analysis of this system plays a central role in this paper, we present a complete derivation of an energy estimate, which will be used later on to prove that energy for the rescaled Einstein equations has the decay property needed for the main result of this paper.

In this section we consider the situation that $\lambda \geq \lambda_{0}$ for some $\lambda_{0}>0$, $\lambda_{0} \neq \frac{(n-1)^{2}}{4 n^{2}}$. Let $-\alpha_{+}$denote the real part of

$$
\frac{-(n-1)+\sqrt{(n-1)^{2}-4 n^{2} \lambda_{0}}}{2},
$$

i.e. $\alpha_{+}=\alpha_{+}\left(n, \lambda_{0}\right)$ is given by

$$
\alpha_{+}=\left\{\begin{array}{cc}
\frac{n-1}{2}, & \lambda_{0}>\frac{(n-1)^{2}}{4 n^{2}} \\
\frac{(n-1)-\sqrt{(n-1)^{2}-4 n^{2} \lambda_{0}}}{2}, & 0<\lambda_{0}<\frac{(n-1)^{2}}{4 n^{2}} .
\end{array}\right.
$$


Define the constant $c_{E}=c_{E}\left(n, \lambda_{0}\right)$ by

$$
c_{E}=\left\{\begin{array}{cc}
\frac{n-1}{2}, & \lambda_{0}>\frac{(n-1)^{2}}{4 n^{2}} \\
\frac{2 n^{2} \lambda_{0}}{(n-1)}, & 0<\lambda_{0}<\frac{(n-1)^{2}}{4 n^{2}} .
\end{array}\right.
$$

Define the energy $E=E\left(X, \dot{X} ; n, \lambda, \lambda_{0}\right)$ by

$$
E=\frac{1}{2} \dot{X}^{2}+\frac{n^{2} \lambda}{2} X^{2}+c_{E} X \dot{X}
$$

and let $X$ be a solution to the damped oscillator equation (6.17) for some $\lambda \geq \lambda_{0}$.

Lemma 6.4. The energy $E$ is positive definite for $\lambda_{0}>0$. Assume $\lambda \geq \lambda_{0}>0$ and $\lambda_{0} \neq \frac{(n-1)^{2}}{4 n^{2}}$. Then $E$ satisfies $\dot{E} \leq-2 \alpha_{+} E$.

Proof. We will consider the case $0<\lambda_{0}<\frac{(n-1)^{2}}{4 n^{2}}$ where anomalous decay holds. The case $\lambda_{0}>\frac{(n-1)^{2}}{4 n^{2}}$ with a universal rate of decay is straightforward and will be left to the reader. The energy $E$ corresponds to the quadratic form

$$
E=\frac{1}{2}\left(\begin{array}{cc}
1 & c_{E} \\
c_{E} & n^{2} \lambda
\end{array}\right)
$$

Let $A=2 E$. In the anomalous case, $c_{E}=2 n^{2} \lambda_{0} /(n-1)$, and setting $Y=4 n^{2} \lambda_{0} /(n-1)^{2}$, we have $0<Y<1$. Then

$$
\begin{gathered}
\operatorname{tr} A=1+n^{2} \lambda, \\
\operatorname{det} A \geq n^{2} \lambda_{0}(1-Y) .
\end{gathered}
$$

Therefore, we have $\operatorname{tr} A>0, \operatorname{det} A>0$ and it follows that $E$ is positive definite.

A calculation shows

$$
\dot{E}=-2 \alpha_{+} E+J
$$

with

$$
\begin{aligned}
J=\left(c_{E}-(n-1)\right. & \left.+\alpha_{+}\right) \dot{X}^{2} \\
& -\left(c_{E}-\alpha_{+}\right) n^{2} \lambda X^{2}-c_{E}\left((n-1)-2 \alpha_{+}\right) X \dot{X},
\end{aligned}
$$

which corresponds to the quadratic form

$$
\left(\begin{array}{cc}
c_{E}-(n-1)+\alpha_{+} & -c_{E}\left((n-1)-2 \alpha_{+}\right) / 2 \\
-c_{E}\left((n-1)-2 \alpha_{+}\right) / 2 & -\left(c_{E}-\alpha_{+}\right) n^{2} \lambda
\end{array}\right)
$$

$J$ is in the anomalous case of the form

$$
J=\frac{n-1}{2}\left(\begin{array}{cc}
Y-1-\sqrt{1-Y} & -\frac{2 n^{2} \lambda_{0}}{n-1} \sqrt{1-Y} \\
-\frac{2 n^{2} \lambda_{0}}{n-1} \sqrt{1-Y} & -n^{2} \lambda[Y-1+\sqrt{1-Y}]
\end{array}\right) .
$$


Setting $B=\frac{2}{n-1} J$, the determinant and trace of $B$ are given by

$$
\begin{aligned}
\operatorname{det} B & =\left(n^{2} \lambda-n^{2} \lambda_{0}\right) Y(1-Y), \\
\operatorname{tr} B & =(Y-1-\sqrt{1-Y})-n^{2} \lambda \sqrt{1-Y}(1-\sqrt{1-Y}) .
\end{aligned}
$$

From this we see that $\operatorname{tr} B<0$, $\operatorname{det} B>0$. Thus, the quadratic form $B$ and hence also $J$ is negative definite. It follows that

$$
\dot{E} \leq-2 \alpha_{+} E
$$

as claimed.

q.e.d.

\section{Energy Estimate}

Taking the analysis in section 6.4 as a guide, we will now define energies for the full Einstein equations. Let the operator $\mathcal{L}_{g, \gamma}$ be given by (6.11). Recall that $\Delta_{g, \gamma}$ as defined in (6.7) is the rough Laplacian on a certain vector bundle $Q$ over $(M, g)$ (see $[\mathbf{2}, \S 2]$ for discussion), which can be identified with the bundle of symmetric covariant 2-tensors on $(M, g)$ with covariant derivative $\nabla[\gamma]$ and fiber metric defined in terms of $\gamma$ by

$$
\langle u, v\rangle=u_{i j} v_{k l} \gamma^{i k} \gamma^{j l}
$$

The corresponding norm is $|u|=(\langle u, u\rangle)^{1 / 2}$. It follows from the definition that the covariant derivative $\nabla[\gamma]$ is metric with respect to $\langle\cdot, \cdot\rangle$. The inner product on derivatives is

$$
\langle\nabla[\gamma] u, \nabla[\gamma] v\rangle=\left\langle\nabla[\gamma]_{m} u, \nabla[\gamma]_{n} v\right\rangle g^{m n} .
$$

The rough Laplacian $\Delta_{g, \gamma}$ is formally self-adjoint with respect to the natural $L^{2}$ inner product

$$
\int_{M}\left\langle u, \Delta_{g, \gamma} v\right\rangle \mu_{g}=\int_{M}\left\langle\Delta_{g, \gamma} u, v\right\rangle \mu_{g}
$$

It follows that the operator $\mathcal{L}_{g, \gamma}$ is self-adjoint with respect to this inner product. We are now able to define the energies which will be used for the Einstein equations. The energy for the damped oscillator consists of a standard oscillator energy and a correction term. Analogously, the energies we are about to define for the rescaled Einstein equations will consist of a wave equation type energy and a correction term. To connect with the damped oscillator energy, we use the correspondence $X \leftrightarrow u$ and $\dot{X} \leftrightarrow v$, where $u=g-\gamma, v=2 n \Sigma$; cf. (6.12).

Throughout the rest of the paper, we fix $s>n / 2+1$, assume that the triple $(\gamma, g, \Sigma)$ satisfies the shadow metric condition, and that $(g, \Sigma) \in$ $B_{\delta, s}(\gamma, 0)$ for some $\delta>0$ sufficiently small. 
The first order energy and correction term is

$$
\begin{aligned}
\mathcal{E}_{(1)} & =\frac{1}{2} \int_{M}|v|^{2} \mu_{g}+\frac{1}{2} n^{2} \int_{M}\left\langle u, \mathcal{L}_{g, \gamma} u\right\rangle \mu_{g}, \\
\Gamma_{(1)} & =\int_{M}\langle v, u\rangle \mu_{g} .
\end{aligned}
$$

Explicitly, substituting in $g-\gamma, \Sigma$ using the relation $u=g-\gamma, v=2 n \Sigma$, this gives

$$
\begin{aligned}
\mathcal{E}_{(1)}= & \frac{1}{2}(2 n)^{2} \int_{M} \Sigma_{i j} \Sigma_{k l} \gamma^{i k} \gamma^{j l} \mu_{g} \\
& +\frac{1}{2} n^{2} \int_{M}\left\{\nabla[\gamma]_{k}\left(g_{i j}-\gamma_{i j}\right) \nabla[\gamma]_{l}\left(g_{m n}-\gamma_{m n}\right) g^{k l} \gamma^{i m} \gamma^{j n}\right. \\
& \left.-2 R[\gamma]_{i j}^{k}{ }_{j}\left(g_{k l}-\gamma_{k l}\right)\left(g_{m n}-\gamma_{m n}\right) \gamma^{i m} \gamma^{j n}\right\} \mu_{g} .
\end{aligned}
$$

The correction term $\Gamma_{(1)}$ can be expanded in a similar manner. We now define higher order energies by inserting suitable powers of $\mathcal{L}_{g, \gamma}$, giving for integers $m \geq 1$,

$$
\begin{aligned}
\mathcal{E}_{(m)} & =\frac{1}{2} \int_{M}\left\langle v, \mathcal{L}_{g, \gamma}^{m-1} v\right\rangle \mu_{g}+\frac{1}{2} n^{2} \int_{M}\left\langle u, \mathcal{L}_{g, \gamma}^{m} u\right\rangle \mu_{g}, \\
\Gamma_{(m)} & =\int_{M}\left\langle v, \mathcal{L}_{g, \gamma}^{m-1} u\right\rangle \mu_{g} .
\end{aligned}
$$

Due to the shadow metric condition, $\gamma$ may be viewed as a function of $g$, and hence the energies $\mathcal{E}_{(s)}$ depend only on $(g, \Sigma)$. In case the lowest eigenvalue of $\mathcal{L}_{\gamma_{0}, \gamma_{0}}$ is zero at the initial background metric, let $\lambda_{\min }^{\prime}>0$ be the smallest nonzero eigenvalue of $\mathcal{L}_{\gamma_{0}, \gamma_{0}}$ and let

$$
\lambda_{0}=\lambda_{\min }^{\prime}-\epsilon
$$

for some $\epsilon>0$. We require $\lambda_{0}>0$. The reason for choosing $\lambda_{0}$ smaller than $\lambda_{\text {min }}^{\prime}$ is that the spectrum of $\mathcal{L}_{\gamma(T)}$ depends on $T$ and it is necessary that the energy estimates we shall prove hold uniformly during the course of the evolution.

Let now $c_{E}, \alpha_{+}$be defined as in section 6.4 in terms of the $\lambda_{0}$ chosen above. For integers $m \geq 1$, let

$$
E_{(m)}=\mathcal{E}_{(m)}+c_{E} \Gamma_{(m)} .
$$

Based on the work in section 6.4, one expects that a corrected energy of the form

$$
E_{s}=\sum_{1 \leq m \leq s} E_{(m)}
$$

will have the property that

$$
\partial_{T} E_{s} \leq-2 \alpha_{+} E_{s}+\text { higher order terms. }
$$

This is indeed the case, as will be shown below. 
Remark 7.1. The value of $\lambda_{0}$ determines $\alpha_{+}$and hence the decay rate that is proved by the present argument. A more detailed analysis, along the lines of [7], can be used to prove a sharp decay estimate.

7.1. Positive definiteness of the energy. Recall that $\mathbb{P}_{\gamma}^{\perp}$, which was introduced in section 2.1, is the $L^{2}$-orthogonal projection onto the orthogonal complement of $\operatorname{ker} \mathcal{L}$ in the space of TT tensors with respect to $\gamma$. It is clear from the construction that for $s>n / 2+1, E_{s}$ is a smooth function on $\mathcal{C}^{s}$ and further that $\mathbb{P}_{\gamma}^{\perp}$ depends smoothly on the Einstein metric $\gamma$.

Lemma 7.2. Let $\gamma$ be an Einstein metric on $M$ with Einstein constant $-(n-1) / n^{2}$ and let $E_{s}$ be the total energy defined in section 7 with $s>n / 2+1$. Then there is $a \delta>0$ and a constant $C>0$, such that for $(g, \Sigma) \in \mathcal{B}_{s, \delta}(\gamma, 0)$, the inequality

$$
\left\|\mathbb{P}_{\gamma}^{\perp}(g-\gamma)\right\|_{H^{s}}^{2}+\|\Sigma\|_{H^{s-1}}^{2} \leq C E_{s}
$$

holds.

Proof. Note that $(\gamma, 0) \in \mathcal{C}^{s}$ is a critical point of $E_{s}$. Therefore, it suffices to consider the second derivative of the energy at $(\gamma, 0)$. Let $m$ be an integer such that $1 \leq m \leq s$. The Hessian of $E_{(m)}$ is of the form

$$
\begin{aligned}
& D^{2} E_{(m)}((h, k),(h, k))= \\
& \quad \int_{M}\left\langle k, \mathcal{L}_{\gamma, \gamma}^{m-1} k\right\rangle \mu_{\gamma}+n^{2} \int_{M}\left\langle h, \mathcal{L}_{\gamma, \gamma}^{m} h\right\rangle \mu_{\gamma}+2 c_{E} \int_{M}\left\langle k, \mathcal{L}_{\gamma, \gamma}^{m-1} h\right\rangle \mu_{\gamma} .
\end{aligned}
$$

An analysis using the spectral decomposition of $\mathcal{L}_{\gamma, \gamma}$ shows, using the arguments in the proof of Lemma 6.4 , that $D^{2} E_{(m)}$ satisfies

$$
D^{2} E_{(m)} \cdot((h, k),(h, k)) \geq 0
$$

with equality if and only if $(h, k)=\left(h^{\mathrm{TT}} \|, 0\right)$ with $h^{\mathrm{TT} \|} \in \operatorname{ker} \mathcal{L}_{\gamma, \gamma}$. From this it follows that

$$
\left\|\mathbb{P}_{\gamma}^{\perp} h\right\|_{H^{s}}^{2}+\|k\|_{H^{s-1}}^{2} \leq C D^{2} E_{s} \cdot((h, k),(h, k))
$$

for some constant $C=C\left(\lambda_{0}, \gamma\right)>0$, where $\lambda_{0}$ is defined in (7.1). It follows from the above and Taylor's theorem that there is a $\delta>0$ such that the inequality (7.3) holds in $\mathcal{B}_{s, \delta}(\gamma, 0)$ for suitable $\delta>0, C>0$. q.e.d.

Lemma 7.3. Let $(\gamma, g, \Sigma)$ be as in Lemma 7.2. There is a $\delta>0$ sufficiently small, and a constant $C>0$, so that if $\|g-\gamma\|_{H^{s}} \leq \delta$,

$$
\left\|\mathbb{P}_{\gamma}^{\|}(g-\gamma)\right\|_{H^{s}} \leq C\left(\left\|\mathbb{P}_{\gamma}^{\perp}(g-\gamma)\right\|_{H^{s}}^{2}+\|\Sigma\|_{H^{s-1}}^{2}\right) .
$$

Proof. By the analysis in section 4.2, we may write

$$
g-\gamma=u^{\mathrm{TT}}+z, \quad \Sigma=v^{\mathrm{TT}}+w
$$


where $u^{\mathrm{TT}}, v^{\mathrm{TT}}$ are TT tensors with respect to $\gamma$ and $z, w$ are $L^{2}$ perpendicular to the space of TT tensors and satisfy

$$
\|z\|_{H^{s}}+\|w\|_{H^{s-1}} \leq C\left(\left\|u^{\mathrm{TT}}\right\|_{H^{s}}^{2}+\left\|v^{\mathrm{TT}}\right\|_{H^{s-1}}^{2}\right)
$$

for $g$ sufficiently close to $\gamma$. Recall that for any TT tensor with respect to $\gamma$,

$$
\left(u^{\mathrm{TT}}, \mathcal{L}_{Y} \gamma\right)_{L^{2}}=0
$$

Using $\mathbb{P}_{\gamma}^{\perp}$, we may split $u^{\mathrm{TT}} L^{2}$-orthogonally as $u^{\mathrm{TT} \|}+u^{\mathrm{TT}} \perp$. Taking equation (4.4) and the just-mentioned facts into account, one finds that equation (4.12) is equivalent to the set of $m$ conditions

$$
\begin{aligned}
0 & =\left(u^{\mathrm{TT}}+z, h^{(\alpha) \mathrm{TT} \|}+\mathcal{L}_{Y^{(\alpha) \|}} \gamma\right)_{L^{2}} \\
& =\left(u^{\mathrm{TT} \|}, h^{(\alpha) \mathrm{TT} \|}\right)_{L^{2}}+\left(z, \mathcal{L}_{Y^{(\alpha) \|}} \gamma\right)_{L^{2}}, \quad \alpha=1, \ldots, m .
\end{aligned}
$$

It follows that this relation defines $u^{\mathrm{TT}} \|$ as a smooth function of $z$, vanishing at $z=0$, and hence in view of (7.4) $z$ is seen to be a function of $u^{\mathrm{TT} \perp}, v^{\mathrm{TT}}$. Since $z$ is of at least second order in $u^{\mathrm{TT}}, v^{\mathrm{TT}}$ and if the shadow relation holds, of $u^{\mathrm{TT} \perp}$, we have

$$
\|z\|_{H^{s}}+\|w\|_{H^{s-1}} \leq C\left(\left\|u^{\mathrm{TT} \perp}\right\|_{H^{s}}^{2}+\left\|v^{\mathrm{TT}}\right\|_{H^{s-1}}^{2}\right) .
$$

The result follows.

q.e.d.

The following result is a direct consequence of Lemmas 7.2 and 7.3, and their proofs.

Theorem 7.4. Suppose that $(\gamma, g, \Sigma)$ satisfy the shadow metric condition, and let $E_{s}$ be the total energy defined in section 7 for $s>n / 2+1$. Then there is $a \delta>0$ and a constant $C>0$, such that for $(g, \Sigma) \in$ $\mathcal{B}_{s, \delta}(\gamma, 0)$, the inequality

$$
\|g-\gamma\|_{H^{s}}^{2}+\|\Sigma\|_{H^{s-1}}^{2} \leq C E_{s}
$$

holds.

We are now able to state the following version of the continuation principle.

Corollary 7.5. Let $\left(\gamma_{0}, g_{0}, \Sigma_{0}\right)$ be an initial data set as in Theorem 7.4, at an initial time $T_{0}$. Let $\left[T_{0}, T_{+}\right)$be the maximal existence interval in $H^{s}, s>n / 2+1$, for the rescaled Einstein equations with the shadow metric condition imposed, with initial data $\left(\gamma_{0}, g_{0}, \Sigma_{0}\right)$.

Then there are numbers $\delta_{0}>0, \delta>0$ so that if $\left(g\left(T_{0}\right), \Sigma\left(T_{0}\right)\right) \in$ $\mathcal{B}_{s, \delta_{0}}\left(\gamma_{0}, 0\right)$ satisfies $E_{s}\left(g\left(T_{0}\right), \Sigma\left(T_{0}\right)\right)<\delta$, then either $T_{+}=\infty$ or there is a finite time $T<\infty$ such that

$$
E_{s}(T) \geq \delta .
$$


This result reduces the problem of proving global existence for the system $(4.9,4.10,4.11)$, with the shadow metric condition (4.12) imposed, to the problem of proving that the energy $E_{s}$ stays small, if it is small initially.

7.2. Time derivative of the energy. We now consider the time derivative of the energy. In order to see the pattern, we do the calculation for the first order energy separately. In the situation considered in Theorem 7.4 and Corollary 7.5, we have

Lemma 7.6. Suppose that $(\gamma, g, \Sigma)$ satisfy the shadow metric condition. There is a $\delta>0$ such that for $(g, \Sigma) \in \mathcal{B}_{s, \delta}(\gamma, 0), s>n / 2+1$, we have

$$
\partial_{T} \mathcal{E}_{(1)}=-(n-1) \int_{M}|v|^{2} \mu_{g}+U_{1}
$$

and

$$
\partial_{T} \Gamma_{(1)} \leq \int_{M}\left(\langle-(n-1) v, u\rangle+|v|^{2}\right) \mu_{g}-n^{2} \int_{M}\left\langle\omega \mathcal{L}_{g, \gamma} u, u\right\rangle \mu_{g}+V_{1}
$$

where

$$
\left|U_{1}\right|+\left|V_{1}\right| \leq C\left(\|g-\gamma\|_{H^{s}}^{3}+\|\Sigma\|_{H^{s-1}}^{3}\right) .
$$

Proof. We have

$$
\begin{aligned}
\partial_{T} \mathcal{E}_{(1)}= & \int_{M}\langle v,-(n-1) v\rangle \mu_{g}-n^{2} \int_{M}\left\langle\mathcal{L}_{g, \gamma} u, h^{\mathrm{TT}} \| \mu_{g}\right. \\
& -\int_{M}\left\langle v, X^{i} \nabla[\gamma]_{i} v\right\rangle \mu_{g}-n^{2} \int_{M}\left\langle\mathcal{L}_{g, \gamma} u, X^{i} \nabla[\gamma]_{i} u\right\rangle \mu_{g}+R_{1}
\end{aligned}
$$

where $R_{1}$ is third order. In particular, using the estimates for $X$, we have

$$
\left|R_{1}\right| \leq C\left(\|u\|_{H^{s}}^{3}+\|v\|_{H^{s-1}}^{3}\right) .
$$

Further, due to the self-adjointness of $\mathcal{L}_{g, \gamma}$ and the fact that $\mathcal{L}_{\gamma, \gamma} h^{\mathrm{TT} \|}=$ 0 , we have that

$$
\mid \int_{M}\left\langle\mathcal{L}_{g, \gamma} u, h^{\mathrm{TT}} \| \mu_{g}\right| \leq C\left(\|u\|_{H^{s}}^{3}+\|v\|_{H^{s-1}}^{3}\right) .
$$

The terms

$$
\begin{aligned}
& \int_{M}\left\langle v, X^{i} \nabla[\gamma]_{i} v\right\rangle \mu_{g}, \\
& \int_{M}\left\langle\mathcal{L}_{g, \gamma} u, X^{i} \nabla[\gamma]_{i} u\right\rangle \mu_{g}
\end{aligned}
$$


also clearly satisfy a third order estimate. Next we consider the correction term. We have, proceeding as above after a direct calculation,

$$
\begin{aligned}
\partial_{T} \Gamma_{(1)}= & \int_{M}\left(\langle-(n-1) v, u\rangle+|v|^{2}\right) \mu_{g}-n^{2} \int_{M}\left\langle\omega \mathcal{L}_{g, \gamma} u, u\right\rangle \mu_{g} \\
& +\int_{M}\left\langle v,(\omega-1) v-h^{\mathrm{TT} \|}\right\rangle \mu_{g} \\
& -\int_{M}\left(\left\langle X^{i} \nabla[\gamma]_{i} v, u\right\rangle+\left\langle v, X^{i} \nabla[\gamma]_{i} u\right\rangle\right) \mu_{g}+S_{1}
\end{aligned}
$$

where $S_{1}$ is third order.

Time differentiating equation (4.12) gives, in view of (6.1),

$$
\begin{aligned}
0 & =\int_{M}\left(g_{i j, T}-\gamma_{i j, T}\right) \gamma^{i k} \gamma^{j l}\left(h_{k l}^{(\alpha) \mathrm{TT} \|}+\mathcal{L}_{Y^{(\alpha)} \|} \gamma_{k l}\right) \mu_{\gamma} \\
& + \text { second order terms } \\
& =\int_{M}\left(g_{i j, T}-h_{i j}^{\mathrm{TT} \|}-\mathcal{L}_{Y \|} \gamma_{i j}\right) \gamma^{i k} \gamma^{j l}\left(h_{k l}^{(\alpha) \mathrm{TT} \|}+\mathcal{L}_{\left.Y^{(\alpha)} \| \gamma_{k l}\right) \mu_{\gamma}}\right. \\
& + \text { second order terms. }
\end{aligned}
$$

Here we have made use of the fact that $\dot{q}^{(\alpha)}$ is of first order. By (6.2), $N / n-1$ is second order. This gives, after using (4.9a) and simplifying,

$$
\begin{aligned}
0 & =\int_{M}\left(2 n \Sigma_{i j}-h_{i j}^{\mathrm{TT} \|}\right) \gamma^{i k} \gamma^{j l}\left(h_{k l}^{(\alpha) \mathrm{TT} \|}+\mathcal{L}_{Y^{(\alpha)} \|} \gamma_{k l}\right) \mu_{\gamma} \\
& + \text { second order terms. }
\end{aligned}
$$

We next note that modulo second order terms, $\Sigma$ is transverse traceless with respect to $\gamma$. This gives

$$
\begin{aligned}
0 & =\int_{M}\left(2 n \Sigma_{i j}-h_{i j}^{\mathrm{TT} \|}\right) h^{(\alpha) \mathrm{TT} \| i j} \mu_{\gamma}+\text { second order terms } \\
& =\int_{M}\left(v_{i j}-h_{i j}^{\mathrm{TT} \|}\right) h^{(\alpha) \mathrm{TT} \| i j} \mu_{\gamma}+\text { second order terms. }
\end{aligned}
$$

We have now proved that

$$
h^{\mathrm{TT}} \|=\mathbb{P}^{\|} v+\text { second order terms }
$$

and hence

$$
\int_{M}\left\langle v, h^{\mathrm{TT} \|}\right\rangle \mu_{g}=\int_{M}\left|v^{\mathrm{TT} \|}\right|^{2} \mu_{g}+\text { third order terms. }
$$

Taking signs into account, we see that

$$
\int_{M}\left\langle v,(\omega-1) v-h^{\mathrm{TT} \|}\right\rangle \mu_{g}=-\int_{M}\left|h^{\mathrm{TT} \|}\right|_{\gamma}^{2} \mu_{\gamma}+\text { third order terms }
$$

can be bounded from above by a third order term. The terms involving $X^{i} \nabla[\gamma]_{i}$ can be handled as above. This completes the proof of the lemma.

q.e.d. 
Since $s>n / 2+1$ is assumed the standard product estimates (cf. e.g. [2, section 2]), allow us to use the fact that we are in a small data situation and handle the higher order terms in the energy in the same way. Let $J_{s}=\sum_{1 \leq j \leq s} \mathcal{J}_{(j)}$ where

$$
\begin{aligned}
\mathcal{J}_{(j)}= & \left(c_{E}-(n-1)+\alpha_{+}\right) \int_{M}\left\langle\mathcal{L}_{g, \gamma}^{j-1} v, v\right\rangle \mu_{g} \\
& -\left(c_{E}-\alpha_{+}\right) n^{2} \int_{M}\left\langle\mathcal{L}_{g, \gamma}^{j} u, u\right\rangle \mu_{g} \\
& -c_{E}\left((n-1)-2 \alpha_{+}\right) \int_{M}\left\langle\mathcal{L}_{g, \gamma}^{j-1} u, v\right\rangle \mu_{g}
\end{aligned}
$$

is defined in analogy with (6.18). An analysis along the lines of Lemma 7.2 , using the estimate for the term $J$ from the proof of Lemma 6.4, shows that the term $J_{s}$ is nonpositive modulo a third order term. This gives

Lemma 7.7. Suppose that $(\gamma, g, \Sigma)$ satisfy the shadow metric condition. There is a $\delta>0$ such that for $(g, \Sigma) \in \mathcal{B}_{s, \delta}(\gamma, 0), s>n / 2+1$, we have

$$
J_{s} \leq C\left(\|g-\gamma\|_{H^{s}}^{3}+\|\Sigma\|_{H^{s-1}}^{3}\right) .
$$

Putting these results together and using Theorem 7.4 gives

Theorem 7.8. Suppose the assumptions of Corollary 7.5 hold. Then, after possibly decreasing $\delta$, there is a constant $C$ such that

$$
\partial_{T} E_{s} \leq-2 \alpha_{+} E_{s}+2 C E_{s}^{3 / 2}
$$

holds if $E_{s}<\delta$.

\section{Future Complete Spacetimes}

In this section, we derive some consequences of the results we have proved for the rescaled Einstein equations. Let $Y=E_{s}^{1 / 2}$ and write $\dot{Y}=\partial_{T} Y$. Then (7.6) takes the form

$$
\dot{Y} \leq-\alpha_{+} Y+C Y^{2} .
$$

The model equation $\dot{y}=-\alpha_{+} y+C y^{2}$ with $y\left(T_{0}\right)=y_{0}>0$ has the solution

$$
y=\frac{\alpha_{+}}{C+e^{\alpha_{+}\left(T-T_{0}\right)}\left[\alpha_{+} / y_{0}-C\right]}
$$

which tends to zero exponentially for $T \geq T_{0}$ if $y_{0}^{-1}>\frac{C}{\alpha_{+}}$. Since $\partial_{T} g$ and hence also $\partial_{T} \gamma$ is bounded in terms of $E_{s}^{1 / 2}$, we see that we can ensure that by starting sufficiently close to data of the form $\left(\gamma_{0}, 0\right) \in \mathcal{N}$, the conditions of Corollary 7.5 remain satisfied for all $T \geq T_{0}$.

We state the conclusion as 
Theorem 8.1. Suppose $\gamma_{0}$ has integrable deformation space. Then there is a $\delta_{1}>0$ such that for any $\left(g_{0}, \Sigma_{0}\right) \in \mathcal{B}_{s, \delta_{1}}\left(\gamma_{0}, 0\right)$, with $\left(\gamma_{0}, g_{0}, \Sigma_{0}\right)$ satisfying the shadow metric condition, the Cauchy problem for the system $(4.9,4.10,4.11)$, with the shadow metric condition imposed, with initial data $\left(\gamma_{0}, g_{0}, \Sigma_{0}\right)$, is globally well posed to the future.

Next we consider the properties of the spacetimes corresponding to the solutions constructed in Theorem 8.1. In order to do this, we rephrase the result in terms of the physical Cauchy data. Thus, let $\left(M, \tilde{g}_{0}, \tilde{K}_{0}\right)$ be CMC vacuum Cauchy data for the Einstein equations with mean curvature $\tau_{0}<0$, and let the spacetime $(\bar{M}, \bar{g})$ be the maximal Cauchy development of $\left(M, \tilde{g}_{0}, \tilde{K}_{0}\right)$. Further, suppose that $\left(M, \tilde{g}_{0}, \tilde{K}_{0}\right)$ is such that for the corresponding rescaled data $\left(g_{0}, \Sigma_{0}\right)$ at scale invariant time $T_{0}$, there exists $\gamma_{0} \in \mathcal{E}_{-(n-1) / n^{2}}$ with integrable deformation space $\mathcal{N}$ and such that the triple $\left(\gamma_{0}, g_{0}, \Sigma_{0}\right)$ is initial data for the rescaled Einstein equations, satisfying the shadow metric condition, with $\left(g_{0}, \Sigma_{0}\right) \in B_{s, \delta_{1}}\left(\gamma_{0}, 0\right)$ for $\delta_{1}$ as in Theorem 8.1. The following corollary to Theorem 8.1 follows by an argument along the lines of [3, $\S 6.1]$.

Corollary 8.2. Let $\left(\gamma_{0}, g_{0}, \Sigma_{0}\right)$ be as in Theorem 8.1, let $\left(\tilde{g}_{0}, \tilde{K}_{0}\right)$ be the corresponding physical Cauchy data, and let $(\bar{M}, \bar{g})$ be the maximal Cauchy development of $\left(\tilde{g}_{0}, \tilde{K}_{0}\right)$. Then

1) The spacetime $(\bar{M}, \bar{g})$ is future complete.

2) The spacetime $(\bar{M}, \bar{g})$ is globally foliated to the future of $(M, \tilde{g}, \tilde{K})$ by CMC Cauchy surfaces, with mean curvature taking all values in $\left[\tau_{0}, 0\right)$.

It follows from the energy estimate that

$$
\|g-\gamma\|_{H^{s}}+\|\Sigma\|_{H^{s-1}} \leq C e^{-\alpha_{+} T}
$$

as $T \rightarrow \infty$, for some constant $C$. Recall that $\gamma$ is the shadow metric of $g$. For the solution curves $T \mapsto(\gamma, g, \Sigma)$ to the rescaled Einstein equations in CMCSH gauge considered in Theorem 8.1, it holds by construction that $\gamma$ stays in a neighborhood of $\gamma_{0}$ in $\mathcal{N}$. In fact, it holds that $\gamma(T)$ tends to a limit in $\mathcal{N}$ as $T \rightarrow \infty$. To see this, we note the following. In view of (6.1), we may estimate $\partial_{T} \gamma$ in terms of $h^{\mathrm{TT}} \|$ and $Y^{\|}$. Equation (2.13) gives an estimate for $Y^{\|}$in terms of $h^{\mathrm{TT}} \|$. Further, equation (7.5) allows us to estimate $h^{\mathrm{TT}} \|$ in terms of $v=2 n \Sigma$ up to terms which are of second order in small quantities. Thus, the inequality (8.1) gives the following corollary to Theorem 8.1.

Corollary 8.3. Let $\left(\gamma_{0}, g_{0}, \Sigma_{0}\right)$ be as in Theorem 8.1, and let $T \mapsto$ $(\gamma, g, \Sigma)$ be the maximal solution to the Cauchy problem for the system $(4.9,4.10,4.11)$, with the shadow metric condition imposed, with initial data $\left(\gamma_{0}, g_{0}, \Sigma_{0}\right)$. Then there is $\gamma_{*} \in \mathcal{N}$ such that $(\gamma, g, \Sigma) \rightarrow\left(\gamma_{*}, \gamma_{*}, 0\right)$ as $T \rightarrow \infty$. 
This shows that there is a limiting Einstein metric for the rescaled Einstein flow, and hence motivates the title of the paper. The above result is completely analogous to the results of $[\mathbf{4}, \mathbf{1 4}]$, which imply that in the case of the $2+1$ dimensional vacuum Einstein equations, the rescaled geometry for a CMC foliation converges to a point in Teichmüller space. Similarly, the conclusion in the work of Moncrief and Choquet-Bruhat [7] is that the conformal geometry of the CMC foliation of the base of the $\mathrm{U}(1)$ bundle converges to a point in Teichmüller space. In the higher dimensional situation considered in the present paper, the Einstein moduli space plays the same role as Teichmüller space.

\section{Appendix A. The Shadow Gauge}

In this section, we let $\mathcal{N}$ be the deformation space with respect to $\gamma_{0}$ and assume that $\mathcal{N}$ is integrable, of dimension $m$. We shall consider some aspects of the shadow gauge condition. We make use of some standard facts from differential topology of infinite dimensional manifolds. All spaces we shall deal with can be viewed either as smooth Hilbert manifolds, modelled on Sobolev spaces $H^{s}$, or as Frechet manifolds, modelled on $C^{\infty}$ viewed as a scale of Sobolev spaces. Further, all maps are smooth with Fredholm Frechet derivatives.

Let $V_{g, \gamma}^{i}$ be the tension field, defined in (2.7). Let $\mathcal{X}$ be the space of vector fields on $M$. We can view $V$ as a map from the Cartesian product of the space of metrics $\mathcal{M}$ with the shadow manifold $\mathcal{N}$ to the space of vector fields,

$$
V: \mathcal{M} \times \mathcal{N} \rightarrow \mathcal{X}
$$

The space of pairs $(g, \gamma) \in \mathcal{M} \times \mathcal{N}$ such that $g$ is in harmonic gauge with respect to $\gamma$ is precisely the zero set of this map. We now shift our attention to the constraint set $\mathcal{C}$. The space $\mathcal{C}$ is a smooth submanifold of the space $T^{\operatorname{tr}} \mathcal{M}$, consisting of pairs $(g, \Sigma)$ satisfying the constraint equations (4.10). By a slight abuse of notation, we can view $V$ as a map $\mathcal{C} \times \mathcal{N} \rightarrow \mathcal{X}$. Let $\mathcal{P}: \mathcal{M} \rightarrow \mathcal{N}$ be the shadow map; cf. remark 4.1. This is a smooth map defined on a neighborhood of $\mathcal{N}$. Similarly to above, we can view $\mathcal{P}$ as defining a map $\mathcal{C} \rightarrow \mathcal{N}$, defined locally near $\left(\gamma_{0}, 0\right)$.

We shall now define a map $\widehat{V}$ on a neighborhood of $\mathcal{N} \times\{0\} \subset \mathcal{C}$, in terms of $V, \mathcal{P}$. We define

$$
\widehat{V}: \mathcal{C} \rightarrow \mathcal{X}, \quad \widehat{V}:(g, \Sigma) \mapsto V_{g, \mathcal{P}(g)} .
$$

We now calculate $\left.D \widehat{V}\right|_{\left(\gamma_{0}, 0\right)}$. We have the decomposition

$$
T_{\left(\gamma_{0}, 0\right)} \mathcal{C}=\left(u^{\mathrm{TT}}+\mathcal{L}_{Y} \gamma_{0}, v^{\mathrm{TT}}\right) .
$$

\section{Lemma A.1.}

$$
\left.D \widehat{V}\right|_{\left(\gamma_{0}, 0\right)} \cdot\left(u^{\mathrm{TT}}+\mathcal{L}_{Y} \gamma_{0}, v^{\mathrm{TT}}\right)=P_{\gamma_{0}, \gamma_{0}} Y,
$$


where the operator $P_{\gamma_{0}, \gamma_{0}}$ is as in (2.8), defined at $(g, \gamma)=\left(\gamma_{0}, \gamma_{0}\right)$.

Proof. We consider the tension field $V$ as a map $(g, \gamma) \rightarrow V_{g, \gamma}$. Let $h=u^{\mathrm{TT}}+\mathcal{L}_{Y} \gamma_{0}$, where $u^{\mathrm{TT}}$ is transverse traceless with respect to $\gamma_{0}$. It follows from $(2.9)$ that $\left.D_{g} V^{i}\right|_{\gamma_{0}, \gamma_{0}} \cdot h=P_{\gamma_{0}, \gamma_{0}} Y^{i}$. Using this together with the fact that $\left.D \mathcal{P}\right|_{\gamma_{0}} \cdot h=u^{\mathrm{TT} \|}$, as follows from equations (6.1) and (2.13), we have

$$
\begin{aligned}
\left.D \widehat{V}^{i}\right|_{\left(\gamma_{0}, 0\right)} \cdot\left(h, v^{\mathrm{TT}}\right) & =\left.D_{g} V^{i}\right|_{\gamma_{0}, \gamma_{0}} \cdot h+\left.\left.D_{\gamma} V^{i}\right|_{\gamma_{0}, \gamma_{0}} \cdot D \mathcal{P}\right|_{\gamma_{0}} \cdot h \\
& =P_{\gamma_{0}, \gamma_{0}} Y^{i}+\left.\gamma_{0}^{m n} D \Gamma_{m n}^{i}\right|_{\gamma_{0}} u^{\mathrm{TT} \|} \\
& =P_{\gamma_{0}, \gamma_{0}} Y^{i}
\end{aligned}
$$

where in the last step we used (4.8).

q.e.d.

As discussed in section 4.2, the operator $P_{\gamma_{0}, \gamma_{0}}$ is an isomorphism. This implies, by an application of the implicit function theorem, the following corollary. Let $\mathcal{S}_{\mathcal{C}, \mathcal{N}}^{\perp} \subset \mathcal{C}$ be the set of solutions to $\widehat{V}=0$, and let $\mathcal{S}_{\mathcal{C}, \gamma}^{\perp}$ be the subset of $\mathcal{S}_{\mathcal{C}, \mathcal{N}}^{\perp}$ consisting of $(g, \Sigma) \in \mathcal{C}$ satisfying the shadow gauge condition with respect to $\gamma$.

Corollary A.2. There is a neighborhood $\mathcal{U}$ of $\left(\gamma_{0}, 0\right) \in \mathcal{C}$ such that $\widehat{V}: \mathcal{U} \rightarrow \mathcal{X}$ is a submersion and

$$
\mathcal{U} \cap \mathcal{S}_{\mathcal{C}, \mathcal{N}}^{\perp}
$$

is a submanifold of $\mathcal{U}$.

Next we consider the action of the diffeomorphism group $\mathcal{D}$. In the applications to $\mathcal{C}$, it is sufficient to work in a neighborhood of $\left(\gamma_{0}, 0\right)$.

\section{Lemma A.3.}

$$
T_{\left(\gamma_{0}, 0\right)} \mathcal{S}_{\mathcal{C}, \mathcal{N}}^{\perp}=T_{\left(\gamma_{0}, 0\right)} \mathcal{S}_{\mathcal{C}, \gamma_{0}}
$$

Proof. Recall from section 4.2 that $T_{\left(\gamma_{0}, 0\right)} \mathcal{S}_{\mathcal{C}, \gamma_{0}}$ is the space $\left\{\left(u^{\mathrm{TT}}, v^{\mathrm{TT}}\right)\right\}$ where $u^{\mathrm{TT}}, v^{\mathrm{TT}}$ are TT tensors with respect to $\gamma_{0}$. This shows, in view of the proof of Lemma A.1, that ker $\left.D \widehat{V}\right|_{\left(\gamma_{0}, 0\right)}=T_{\left(\gamma_{0}, 0\right)} \mathcal{S}_{\mathcal{C}, \gamma_{0}}$, and hence $T_{\left(\gamma_{0}, 0\right)} \mathcal{S}_{\mathcal{C}, \mathcal{N}}^{\perp}=T_{\left(\gamma_{0}, 0\right)} \mathcal{S}_{\mathcal{C}, \gamma_{0}}$ as claimed.

q.e.d.

Taking Lemma A.3 into account, we can now complete the analysis of the shadow gauge condition.

Proposition A.4. There is a neighborhood $\mathcal{U}$ of $\left(\gamma_{0}, 0\right) \in \mathcal{C}$ such that for $(g, \Sigma) \in \mathcal{U}$, there is a unique $\phi \in \mathcal{D}$, so that

$$
\left(\phi^{*} g, \phi^{*} \Sigma\right) \in \mathcal{S}_{\mathcal{C}, \mathcal{N}}^{\perp} .
$$

In particular, for $(g, \Sigma) \in \mathcal{U}$, there are unique $\phi \in \mathcal{D}, \gamma \in \mathcal{N}$, such that $\left(\phi^{*} g, \phi^{*} \Sigma\right) \in \mathcal{S}_{\mathcal{C}, \gamma}^{\perp}$, i.e. such that $\left(\phi^{*} g, \phi^{*} \Sigma, \gamma\right)$ satisfy the shadow gauge condition. 
Proof. We show that the map $L: \mathcal{D} \times \mathcal{S}_{\mathcal{C}, \mathcal{N}}^{\perp} \rightarrow \mathcal{C}$, defined by

$$
L(\phi, g, \Sigma)=\left(\phi^{*} g, \phi^{*} \Sigma\right),
$$

is a diffeomorphism locally at $\left(\mathbf{i},\left(\gamma_{0}, 0\right)\right)$, where $\mathbf{i}$ denotes the identity in $\mathcal{D}$. Write a general element in $T_{\mathbf{i}} \mathcal{D}$ as $X$, and a general element of $T_{\left(\gamma_{0}, 0\right)} \mathcal{S}_{\mathcal{C}, \mathcal{N}}^{\perp}$ as $\left(u^{\mathrm{TT}}, v^{\mathrm{TT}}\right)$. We have

$$
\left.D L\right|_{\left(\mathbf{i},\left(\gamma_{0}, 0\right)\right)}=\left(u^{\mathrm{TT}}+\mathcal{L}_{X} \gamma_{0}, v^{\mathrm{TT}}\right) .
$$

Recalling that $T_{\left(\gamma_{0}, 0\right)} \mathcal{C}$ is spanned by tensors of the form $\left(u^{\mathrm{TT}}+\mathcal{L}_{X} \gamma_{0}\right.$, $v^{\mathrm{TT}}$ ), we see that the Frechet derivative $D L$ is an isomorphism

$$
\left.D L\right|_{\left(\mathbf{i},\left(\gamma_{0}, 0\right)\right)}: T_{\mathbf{i}} \mathcal{D} \times T_{\left(\gamma_{0}, 0\right)} \mathcal{S}_{\mathcal{C}, \mathcal{N}}^{\perp} \rightarrow T_{\left(\gamma_{0}, 0\right)} \mathcal{C},
$$

and the proposition follows.

q.e.d.

Proposition A.4 establishes the validity of Lemma 4.3.

\section{References}

1. Lars Andersson, Thierry Barbot, Riccardo Benedetti, Francesco Bonsante, William M. Goldman, François Labourie, Kevin P. Scannell, \& Jean-Marc Schlenker, Notes on: "Lorentz spacetimes of constant curvature" [Geom. Dedicata 126 (2007), 3-45] by G. Mess, Geom. Dedicata 126 (2007), 47-70.

2. Lars Andersson \& Vincent Moncrief, Elliptic-hyperbolic systems and the Einstein equations, Ann. Henri Poincaré 4 (2003), no. 1, 1-34.

3. - Future complete vacuum spacetimes, The Einstein equations and the large scale behavior of gravitational fields, Birkhäuser, Basel, 2004, pp. 299-330.

4. Lars Andersson, Vincent Moncrief, \& Anthony J. Tromba, On the global evolution problem in $2+1$ gravity, J. Geom. Phys. 23 (1997), no. 3-4, 191-205.

5. Arthur L. Besse, Einstein manifolds, Springer-Verlag, Berlin, 1987.

6. G. Besson, G. Courtois, \& S. Gallot, Entropies et rigidités des espaces localement symétriques de courbure strictement négative, Geom. Funct. Anal. 5 (1995), no. 5, 731-799.

7. Yvonne Choquet-Bruhat \& Vincent Moncrief, Future global in time Einsteinian spacetimes with U(1) isometry group, Ann. Henri Poincaré 2 (2001), no. 6, 10071064 .

8. Xianzhe Dai, Xiaodong Wang, \& Guofang Wei, On the variational stability of Kähler-Einstein metrics, Comm. Anal. Geom. 15 (2007), no. 4, 669-693.

9. Dennis M. DeTurck \& Jerry L. Kazdan, Some regularity theorems in Riemannian geometry, Ann. Sci. École Norm. Sup. (4) 14 (1981), no. 3, 249-260.

10. Arthur E. Fischer \& Vincent Moncrief, Hamiltonian reduction and perturbations of continuously self-similar $(n+1)$-dimensional Einstein vacuum spacetimes, Classical Quantum Gravity 19 (2002), no. 21, 5557-5589.

11. Michael Kapovich, Deformations of representations of discrete subgroups of SO(3,1), Math. Ann. 299 (1994), no. 2, 341-354.

12. Claude LeBrun, Einstein metrics and Mostow rigidity, Math. Res. Lett. 2 (1995), no. $1,1-8$. 
13. Geoffrey Mess, Lorentz spacetimes of constant curvature, Geom. Dedicata 126 (2007), 3-45.

14. Vincent Moncrief, Relativistic Teichmüller theory-a Hamilton-Jacobi approach to 2+1-dimensional Einstein gravity, Surveys in differential geometry. Vol. XII. Geometric flows, Surv. Differ. Geom., vol. 12, Int. Press, Somerville, MA, 2008, pp. 203-249.

15. Martin Reiris, On the asymptotic spectrum of the reduced volume in cosmological solutions of the Einstein equations, Gen. Relativity Gravitation 41 (2009), no. 5, 1083-1106.

16. José M. M. Senovilla, The universal 'energy' operator, Classical Quantum Gravity 23 (2006), no. 23, 7143-7147.

Department of Mathematics

UNIVERSITY OF MIAMI

Coral Gables, FL 33124

AND

Albert Einstein Institute

AM MühlenBerg 1

D-14476 PoTSDAM

Germany

E-mail address: laan@aei.mpg.de

Department of Physics and Department of Mathematics

YALE UNIVERSity

P.O. Box 208120

New Haven, CT 06520

E-mail address: vincent.moncrief@yale.edu 